\title{
Spontaneous tubulation of membranes and vesicles reveals membrane tension generated by spontaneous curvature $\dagger$
}

\author{
Reinhard Lipowsky
}

\author{
Received 9th May 2012, Accepted 9th July 2012 \\ DOI: $10.1039 / c 2 f d 20105 d$
}

\begin{abstract}
Recent experimental studies on supported lipid bilayers and giant vesicles have shown that uni-lamellar membrane systems can undergo spontaneous tubulation, i.e., can form membrane tubules or nanotubes without the application of external forces. In the case of supported lipid bilayers, the tube formation was induced by the adsorption of antimicrobial peptides. In the case of giant vesicles, spontaneous tubulation was observed after the polymer solution inside the vesicles underwent phase separation into two aqueous phases. Here, these processes are studied theoretically and shown to be driven by membrane tension generated by spontaneous curvature. The latter curvature is estimated for different types of adsorbing particles, such as ions, small molecules, and macromolecules, that differ in their size and in their adsorption kinetics. When the two sides of the membranes are exposed to two different concentrations of these particles, the membranes will acquire a spontaneous (or preferred) curvature. Particularly large spontaneous curvatures are induced by the adsorption of amphipathic peptides and BAR domain proteins. Another mechanism that induces spontaneous curvature is provided by different depletion layers in front of the two sides of the membranes. Irrespective of its molecular origin, a spontaneous curvature is predicted to generate a tension in weakly curved membranes, a 'spontaneous' tension that can vary over several orders of magnitudes and can be as high as $1 \mathrm{~mJ} \mathrm{~m}^{-2}$. The concept of spontaneous tension is first used to explain the spontaneous tubulation of supported lipid bilayers when exposed to adsorbing particles. This tubulation process is energetically preferred when the spontaneous tension exceeds the adhesive strength of the underlying solid support. Furthermore, in the case of giant vesicles, the spontaneous tension can balance the osmotic pressure difference between the interior and exterior aqueous compartment. The vesicles are then able to form stable cylindrical nanotubes that protrude into the vesicle interior as observed recently for membranes in contact with two aqueous polymer phases. In these latter systems, the vesicle membranes are governed by two spontaneous tensions that can be directly measured since they are intimately related to the effective and intrinsic contact angles.
\end{abstract}

Theory and Bio-Systems, Max Planck Institute of Colloids and Interfaces, 14424 Potsdam, Germany.E-mail: lipowsky@mpikg.mpg.de; Fax: +49 331567 9602; Tel: +49 3315679600

$\dagger$ Electronic Supplementary Information (ESI) available with computational details: Appendix A on 'Spontaneous curvature induced by adsorption' and Appendix B on 'Stress-free shapes of spherical and cylindrical membranes'. See DOI: 10.1039/c2fd20105d 


\section{Introduction}

Membranes and vesicles often attain spherical shapes or undergo shape transformations that lead to spherical buds. Such shapes have been studied for several decades, both experimentally and theoretically, and are reasonably well understood. It is also known for some time that membrane tubules or nanotubes, often denoted as "tethers", can be pulled from large vesicles by applying external forces to the membranes. It is less obvious that such tubules may also form spontaneously, i.e., in the absence of external forces. Nevertheless, spontaneous tubulation processes have been observed for a variety of systems.

About a hundred years ago, tubular 'myelin' structures were observed by optical microscopy when lamellar liquid crystals of ammonium oleate or lecithin were swollen in excess water, see Fig. 1. ${ }^{1}$ More recently, many different proteins have been identified that adsorb onto multilamellar liposomes and lead to extended membrane tubules. These proteins include N-BAR proteins, such as amphiphysin ${ }^{2,3}$ and endophilin, ${ }^{4}$ F-BAR proteins, such as syndapins, ${ }^{5}$ and other proteins involved in endocytosis, such as epsin. ${ }^{6}$

Very recently, two different unilamellar systems have been found to undergo spontaneous tubulation. One of these systems is provided by supported lipid bilayers that were exposed to a variety of antimicrobial peptides..$^{7-10}$ The other system consists of giant vesicles in contact with aqueous solutions of PEG and dextran that undergo phase separation into a PEG-rich and a dextran-rich phase. ${ }^{11}$

In this paper, the process of spontaneous tubulation is studied theoretically in order to develop a unified perspective for all of these systems. First, different species of adsorbing particles are considered, such as ions, small molecules, and macromolecules, which differ in their size and in their adsorption kinetics. When the two sides of the membranes are exposed to different concentrations of the adsorbing particles, their adsorbate coverage will be different, and the asymmetric membranes will then prefer to bend to one of the aqueous compartments. In all examples considered here, the membranes prefer to bend away from the compartment with the higher concentration of adsorbing particles. ${ }^{12}$ This asymmetry is quantitatively described by the spontaneous (or preferred) curvature of the membranes. Another mechanism that

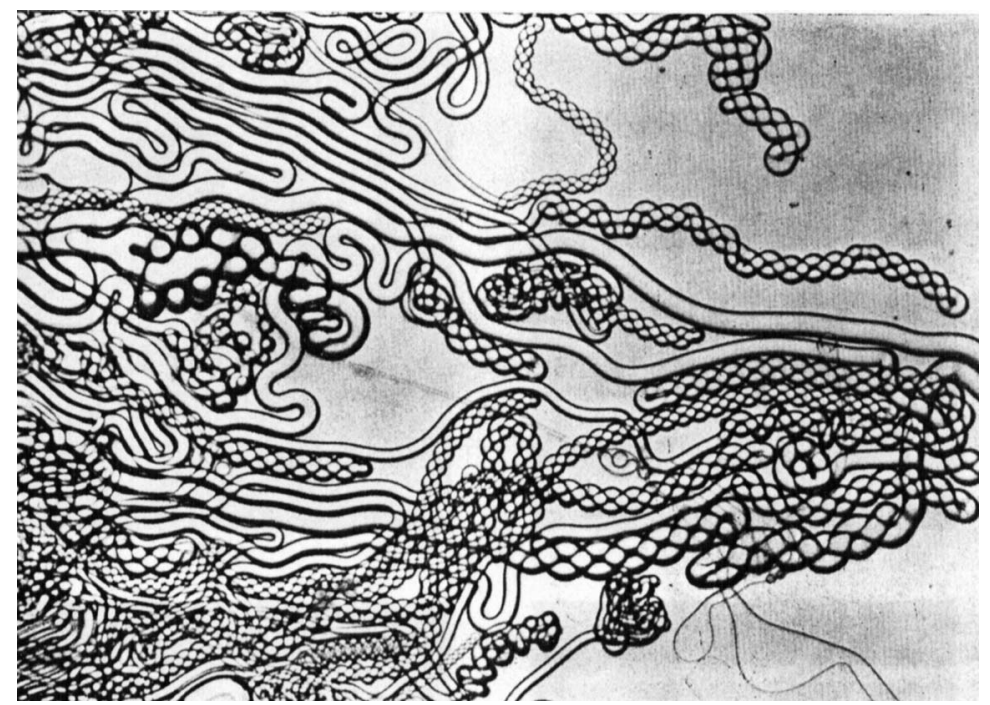

Fig. 1 Tubular structures as observed by Otto Lehmann about a hundred years ago when lamellar liquid crystals of ammonia oleate were exposed to excess water. ${ }^{1}$ 
induces spontaneous curvature is provided by different depletion layers in front of the two membrane surfaces. ${ }^{12}$ Irrespective of its molecular origin, a spontaneous curvature is predicted to generate a membrane tension that can vary over a wide range and can be as large as $1 \mathrm{~mJ} \mathrm{~m}^{-2}$. Since this tension is intimately related to the spontaneous curvature, the term 'spontaneous tension' as introduced here seems appropriate for it.

The spontaneous tension is shown to be a rather useful concept in order to understand the tubulation of supported lipid bilayers as observed experimentally in ref. 7-10. Indeed, spontaneous tubulation of the bilayer is predicted to depend on the relative magnitude of the adsorption-induced spontaneous tension and the adhesive strength of the underlying substrate. Thus, this tubulation process can be understood as the competition between the adhesive support and the adsorbing particles. A similar competition should also apply to the outmost membrane of a liposome, when this membrane acquires a spontaneous curvature via adsorption.

The concept of spontaneous tension also provides useful insight into the spontaneous tubulation of giant vesicles as observed in ref. 11 . When viewed with optical microscopy, the shapes of these vesicles consist of two spherical caps and, thus, appear to be rather tense. As shown here, this shape is primarily governed by the spontaneous tension generated by the spontaneous curvature. In fact, the spherical caps are described, to an excellent approximation, by simple Laplace-like equations, in which the osmotic pressure differences are balanced by the spontaneous tensions alone. The latter tensions are also shown to be intimately related to the effective and intrinsic contact angles that can be determined directly from the optical micrographs.

This article is organized as follows: section 2 describes the theoretical approach, which is based on a mesoscopic description of the membranes in terms of their elastic properties. For weakly curved membranes, the main contribution to the bending energy arises from the spontaneous tension generated by the spontaneous curvature. Section 3 determines the spontaneous curvature induced by the adsorption of a variety of particles, such as ions, small molecules, as well as polymers and proteins. The latter section also describes the range of values that the spontaneous curvatures and tensions can assume. Supported lipid bilayers that acquire a spontaneous curvature by adsorption are considered in section 4 , where a simple criterion for spontaneous tubulation is derived. Giant vesicles bounded by membranes with a relatively large spontaneous curvature are discussed in section 5 and giant vesicles in contact with two aqueous phases in section 6 . The last section contains a brief summary and outlook.

\section{Theoretical preliminaries}

The theoretical description used here is based on a mesoscopic view of the membranes. Indeed, the membranes will be primarily characterized by their surface area $A$ and two elastic parameters, their bending rigidity $\kappa$, which provides the basic energy scale, and their spontaneous (or preferred) curvature $m$, which represents an inverse length scale. The bending rigidity $\kappa$ was originally introduced in the context of elastic membrane models for giant vesicles ${ }^{13-15}$ but is, in fact, a meaningful concept down to molecular scales as revealed by molecular dynamics simulations. ${ }^{16}$ The spontaneous curvature $m$ is a phenomenological parameter for the intuitive notion that a surface may prefer to bend towards one side rather than towards the other. It was originally considered for monolayers by Bancroft and Tucker ${ }^{17}$ and introduced for bilayers by Helfrich. ${ }^{14}$

If flip flops between the two leaflets of the bilayers are sufficiently slow, the elastic energy also contains a contribution from the area difference between these two leaflets governed by another bending rigidity, ${ }^{18}$ which can be easily incorporated into the theory but is omitted here in order to eliminate one parameter from the discussion. 


\subsection{Sign convention about the membrane's mean curvature}

All theories that describe the membrane's bending energy involve the mean curvature $M$, which is equal, for each point on the membrane surface to the arithmetic mean of the two principal curvatures $C_{1}$ and $C_{2}$ at this point, i.e., $M=\frac{1}{2}\left(C_{1}+C_{2}\right)$.

It is important to realize that the mean curvature $M$ may be positive or negative since each membrane has two sides or surfaces, which are in contact with two distinct aqueous compartments, the exterior and the interior one, see Fig. 2. For a closed vesicle or cell membrane, the interior compartment is the one enclosed by the membrane. For a supported bilayer, the interior compartment is bounded by the bilayer membrane and the solid substrate surface. Here and below, I will use the sign convention that the mean curvature $M$ is positive if the membrane bends locally away from its exterior surface, i.e., bulges locally towards the exterior compartment, see Fig. 2(a). Likewise, $M$ is negative if the membrane bends locally away from its interior surface.

The sign convention for $M$ is intimately related to the choice for the normal vector of the membrane surface. The convention used here implies that this normal vector is always chosen to point into the exterior compartment.

\subsection{Bending energy of the membrane}

If the membrane has a uniform composition, its shape is governed by uniform elastic properties as described by constant bending rigidity $\kappa$ and spontaneous curvature $m$ \$ Following the sign convention for the mean curvature, see Fig. 2, the spontaneous curvature $m$ is taken to be positive and negative if the membrane prefers to bend away from (or bulge towards) the exterior and interior compartment, respectively. The membrane shape is then governed by the bending energy

$$
\mathscr{E}_{\mathrm{be}}=\int d A 2 \kappa(M-m)^{2},
$$

which represents an integral over the membrane area and depends on the mean curvature $M$ that will, in general, vary along the membrane. Furthermore, I will consider membrane processes at constant temperature, for which the membrane area $A$ remains constant on the experimentally relevant time scales.

The bending energy (1) vanishes for $M=m$, i.e., if the whole membrane adapts its shape to the spontaneous curvature $m$. In general, such an adaptation will not be possible, however, since the membrane experiences additional constraints arising, e.g., from adhesive surfaces or osmotic conditions.

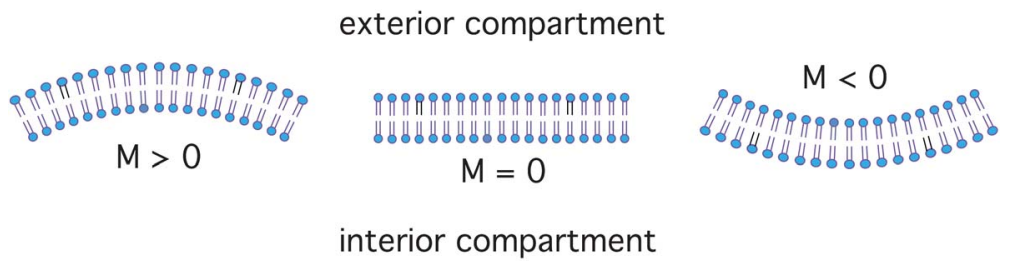

(a)

(b)

Fig. 2 Sign convention for mean curvature $M$ : (a) The mean curvature is positive if the membrane bends locally away from (or bulges locally towards) the exterior compartment; (b) the mean curvature vanishes for a planar membrane; and (c) the mean curvature is negative if the membrane bends locally away from (or bulges towards) the interior compartment.

$\$$ The term 'spontaneous curvature' is used in this paper as an abbreviation for 'spontaneous mean curvature', which explains the notation $m$. 


\subsection{Definition of spontaneous tension}

Now, assume that a large segment of the membrane cannot attain the optimal mean curvature $M=m$. It is then instructive to rewrite the bending energy (1) in the form:

$$
\mathscr{E}_{\mathrm{be}}=2 \kappa m^{2} A-4 \kappa m r_{1}+2 \kappa r_{2},
$$

with

$$
r_{1} \equiv \int \mathrm{d} A M \text { and } r_{2} \equiv \int \mathrm{d} A M^{2}
$$

The total mean curvature $r_{1}$ has the dimension of a length and the integral $r_{2}$ is dimensionless. For a sphere with radius $R_{\mathrm{sp}}$, for instance, one has $r_{1}=4 \pi R_{\mathrm{sp}}$ and $r_{2}=4 \pi$. Therefore, when a large membrane segment of area $\Delta A$ cannot adapt to its optimal mean curvature $M=m$, the bending energy behaves as

$$
\mathscr{E}_{\mathrm{be}} \approx 2 \kappa m^{2} \Delta A \text { for large } \Delta A,
$$

which is, in fact, the bending energy of a planar membrane with $M=0$ and spontaneous curvature $m$. Therefore, a planar or weakly curved membrane with mean curvature $|M| \ll|m|$ experiences the spontaneous tension

$$
\sigma \equiv 2 \kappa m^{2}
$$

In the next section, we will see that the magnitude of this tension can vary over a wide range, from $10^{-6} \mathrm{~mJ} \mathrm{~m}^{-2}$ up to $1 \mathrm{~mJ} \mathrm{~m}^{-2}$. Furthermore, the spontaneous tension $\sigma$ will play an important role in all subsequent sections since it turns out to be the main driving force for the spontaneous tubulation of membranes.

\section{Spontaneous curvature induced by adsorption processes}

In general, one can envisage a variety of molecular mechanisms for the generation of spontaneous (or preferred) curvature. Here, I will focus on mechanisms related to the adsorption of ions, small molecules, and macromolecules, such as polymers and proteins, from the surrounding aqueous solution. In general, adsorption can take place on both membrane sides or surfaces. Since the exterior and interior membrane surface are exposed to different aqueous compartments, the corresponding adsorption processes may lead to different ionic or molecular compositions of the two surfaces. In such a situation, the membrane will prefer to bend away from one of the compartments and will, thus, acquire a spontaneous (or preferred) curvature. As shown in ref. 12, the Gibbs adsorption equation implies that the membrane will bend away from the compartment that leads to the larger coverage of adsorbed ions or small molecules.

In the next three subsections, the spontaneous curvature will be estimated for three different adsorption processes: (i) adsorption of ions and small molecules; (ii) adsorption of flexible polymers; and (iii) adsorption of BAR domain proteins. The last subsection describes the range of values that the spontaneous curvature and, thus, the spontaneous tension may assume.

\subsection{Spontaneous curvature from adsorbed ions and small molecules}

First, consider a membrane in contact with aqueous compartments that contain small 'particles', such as atomic ions or small molecules, which adsorb onto the membrane surfaces. The particles are osmotically active in the sense that the membrane is impermeable to these particles on the relevant time scales. Furthermore, in this subsection, the particles are taken to be small compared to the membrane thickness of about $4 \mathrm{~nm}$. In such a situation, the membrane prefers to 
bend away from the membrane surface with the larger coverage of adsorbed particles as shown in Fig. 3(a). ${ }^{12}$

The value of the spontaneous curvature can be estimated if one views the membrane as an ultra-thin film bounded by two surfaces that are exposed to the particle concentrations $C_{\mathrm{ex}}$ and $C_{\mathrm{in}}$ in the exterior and interior compartment, respectively. ${ }^{12,19}$ Different concentrations lead to different coverages $\Gamma_{\text {ex }}$ and $\Gamma_{\text {in }}$ of the two membrane surfaces. As shown in Appendix A in the ESI, $\dagger$ a membrane of thickness $\ell_{\text {me }}$ and bending rigidity $\kappa$ is theoretically found to exhibit the spontaneous curvature:

$$
m=\frac{k_{\mathrm{B}} T}{4 \kappa} \ell_{\mathrm{me}}\left(\Gamma_{\mathrm{ex}}-\Gamma_{\mathrm{in}}\right)
$$

for dilute solutions of the adsorbing particles. Thus, the spontaneous curvature $m$ is proportional to the dimensionless ratio $k_{\mathrm{B}} T / \kappa$, to the membrane thickness $\ell_{\text {me }} \simeq 4$ $\mathrm{nm}$, and to the coverage difference $\Gamma_{\mathrm{ex}}-\Gamma_{\mathrm{in}}$. The coverages can be parametrized in terms of the maximal coverage $\Gamma_{\max }$, see eqn (A.3) in the ESI, $\uparrow$ and the equilibrium constant $K_{\mathrm{d}}$, see eqn (A.6) in the ESI. $\dagger$ One then obtains:

$$
m=\frac{k_{\mathrm{B}} T}{4 \kappa} \ell_{\mathrm{me}} \Gamma_{\max } \frac{C_{\mathrm{ex}}-C_{\mathrm{in}}}{K_{\mathrm{d}}}
$$

for small concentrations $C_{\mathrm{ex}} \lesssim K_{\mathrm{d}}$ and $C_{\text {in }} \lesssim K_{\mathrm{d}}$. For lipid bilayers, the ratio $k_{\mathrm{B}} T / \kappa \simeq$ $1 / 20$. The inverse length scale $\ell_{\mathrm{me}} \Gamma_{\max } / 4$ is related to the molecular dimensions of the adsorbed particles and the surface density of binding sites, see eqn (A.3) in the ESI. $\dagger$ If the maximal coverage is of the order of one adsorbed particle per head group area, one has $\ell_{\mathrm{me}} \Gamma_{\mathrm{max}} / 4 \simeq 1 \mathrm{~nm}^{-1}$. In such a situation, the adsorbed particles can induce a spontaneous curvature $m$ up to about $1 /(20 \mathrm{~nm})$ for $C_{\mathrm{ex}} \simeq K_{\mathrm{d}}$ and $C_{\mathrm{in}}=0$.

The sign of the spontaneous curvature $m$ is determined by the sign of $\Gamma_{\mathrm{ex}}-\Gamma_{\mathrm{in}} \propto$ $C_{\mathrm{ex}}-C_{\mathrm{in}}$. Thus, $m$ is positive and bends away from the exterior compartment if the concentration $C_{\mathrm{ex}}$ in this compartment exceeds the concentration $C_{\mathrm{in}}$ in the interior compartment. This distinction also applies to the examples discussed in the following subsections. However, in order to simplify the notation, I will now focus on the limiting case, in which $C_{\text {in }}=0$ and the particles adsorb only from the exterior compartment, see Fig. 3.

\subsection{Adsorption of flexible polymers}

As a second mechanism, consider flexible polymers, such as polypeptides or DNA strands, that are adsorbed by membrane-anchored molecules. The size of the polymers as measured by their average end-to-end distance, $R_{\mathrm{po}}$, is now taken to be large

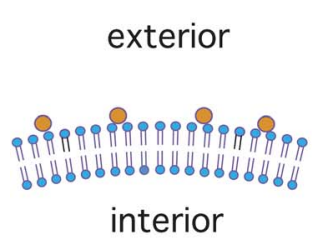

(a)

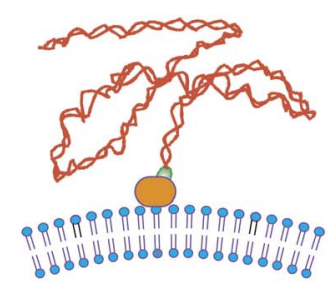

(b)

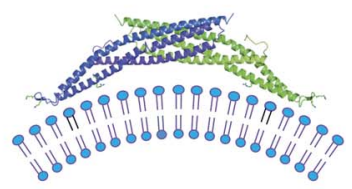

(c)

Fig. 3 Membrane curvature generated by adsorption from the exterior compartment: (a) adsorption of ions or small molecules (orange): the membrane curves towards the adsorbate; ${ }^{12}$ (b) adsorption of flexible polymers to membrane anchors, ${ }^{20}$ such as biotinylated DNA (red) to membrane-anchored avidin (orange); ${ }^{21}$ and (c) adsorption of N-BAR domain proteins that mold the local membrane shape., ${ }^{3,22}$ The thickness of the bilayer membranes (blue) is about $4 \mathrm{~nm}$. 
compared to the membrane thickness. An example is provided by biotinylated DNA strands that are adsorbed to a membrane-anchored avidin molecules, ${ }^{21}$ see Fig. 3(b). When the flexible polymer is adsorbed, it loses configurational entropy that acts to bend the membrane away from it. More precisely, the entropy loss of a single polymer with average end-to-end distance $R_{\mathrm{po}}$ induces the spontaneous curvature: ${ }^{20}$

$$
m_{1}=\frac{q}{\pi} \frac{k_{\mathrm{B}} T}{4 \kappa} \frac{1}{R_{\mathrm{po}}}
$$

within a membrane segment of area $A_{\mathrm{po}}=\pi R_{\mathrm{po}}^{2}$, where the dimensionless coefficient $q$ is equal to $(\pi / 6)^{1 / 2}$ for ideal (or Gaussian) polymer chains and of order one in general. Thus, the integrated mean curvature induced by a single polymer is $A_{\mathrm{po}} m_{1}$. Furthermore, if $N_{\text {po }}$ polymers are adsorbed onto the exterior membrane surface with area $A$, the polymer coverage of this surface is equal to $\Gamma_{\mathrm{po}}=N_{\mathrm{po}} / A$. The entropy loss of the adsorbed polymers now induces the spontaneous curvature: ${ }^{23}$

$$
m=A_{\mathrm{po}} m_{1} \Gamma_{\mathrm{po}}=q \frac{k_{\mathrm{B}} T}{4 \kappa} R_{\mathrm{po}} \Gamma_{\mathrm{po}},
$$

as obtained from a superposition of the curvatures induced by the individual polymers. Compared to eqn (6) for ions and small molecules, the membrane thickness $\ell_{\text {me }}$ has now been replaced by the polymer size $R_{\text {po }}$. The maximal coverage $\Gamma_{\max }$ that can be obtained by adsorption of water-soluble polymers should be of the order of $1 / R_{\mathrm{po}}^{2}$. For this coverage, the spontaneous mean curvature $m$ is of the order of $k_{\mathrm{B}} T /\left(4 \kappa R_{\mathrm{po}}\right)$ as in eqn $(8)$, which is about $1 /\left(80 R_{\mathrm{po}}\right)$ for lipid bilayers with $k_{\mathrm{B}} T / \kappa \simeq 1 / 20$.

When the polymer coverage $\Gamma_{\text {po }}$ is expressed in terms of the exterior concentration $C_{\text {po }}$ and the equilibrium constant $K_{\mathrm{d}}$, one obtains the expression:

$$
m=q \frac{k_{\mathrm{B}} T}{4 \kappa} \frac{1}{R_{\mathrm{po}}} \frac{C_{\mathrm{po}}}{K_{\mathrm{d}}} \text { for } C_{\mathrm{po}} \lesssim K_{\mathrm{d}},
$$

where $\Gamma_{\max }=1 / R_{\mathrm{po}}^{2}$ has been used.

For DNA anchored to DOPC membranes, as experimentally studied in ref. 21, the spontaneous curvature $m$ has been estimated from vesicle budding. Indeed, for a membrane with uniform composition, the mean curvatures $M_{\mathrm{ve}}$ and $M_{\text {bud }}$ of mother vesicle and bud close to their joint neck determine the spontaneous curvature via the neck condition: $:^{24,25}$

$$
m=\frac{1}{2}\left(M_{\mathrm{ve}}+M_{\mathrm{bud}}\right) .
$$

The spontaneous curvature $m$ deduced in this manner was found to be of the order of $1 \mu \mathrm{m}^{-1}$. On the other hand, the entropy loss of the anchored polymers should induce the curvature $m \simeq 1 /\left(80 R_{\mathrm{po}}\right)$ which is about $\left.1 / 52 \mu \mathrm{m}\right)$ since the DNA strands used in ref. 21 had the size $R_{\mathrm{po}} \simeq 0.65 \mu \mathrm{m}$. One simple explanation for the much larger experimental value of $m$ is provided by the putative adsorption of counterions onto the membrane. Indeed, the negatively charged DNA attracts a cloud of positively charged counterions and the adsorbed DNA drags this cloud towards the membrane, which implies an increased counterion concentration close to the membrane. If these ions adsorb onto the membrane with the relatively small coverage $\Gamma_{\mathrm{ex}} \simeq 1 /\left(50 \mathrm{~nm}^{2}\right)$, this adsorption process would induce the spontaneous curvature $m \simeq 1 \mu \mathrm{m}^{-1}$ according to eqn (6).

\subsection{Adsorption of BAR domain proteins}

During the last decade, many proteins have been found that have a banana-like shape and bind to membranes, thereby molding the shape of these membranes 
and generating an appreciable membrane curvature. In fact, a whole superfamily of BAR domain proteins has been identified, that include N-BAR proteins, such as amphiphysin $^{2,3}$ and endophilin, ${ }^{4}$ as well as F-BAR proteins, such as syndapins ${ }^{5}$ (for reviews, see ref. 22,26-28). All of these molecules are banana-shaped protein dimers with a concave, positively charged surface that binds to negatively charged membranes.

As an example, let us consider the amphiphysin N-BAR protein depicted in Fig. 3(c). ${ }^{3,22}$ The banana-like shape of this protein has a membrane binding surface with a length of about $14.4 \mathrm{~nm}$, a width of about $1.6 \mathrm{~nm}$, and a curvature radius $R_{\mathrm{BAR}}$ of about $11 \mathrm{~nm} .^{3}$ Thus, it covers a membrane area $A_{\mathrm{BAR}}$ of about $23 \mathrm{~nm}^{2}$.

It is believed that a single BAR domain protein can bend the adjacent membrane segment of area $A_{\mathrm{BAR}}$ in such a way that this membrane segment acquires the mean curvature:

$$
M=m_{1}=1 /\left(2 R_{\mathrm{BAR}}\right) .
$$

Such a process involves the bending energy:

$$
\mathscr{E}_{\mathrm{be}}=A_{\mathrm{BAR}} 2 \kappa m_{1}^{2} \simeq \kappa / 11,
$$

where the latter estimate corresponds to the amphiphysin N-BAR protein. This bending energy must be overcome by the energy gain arising from adhesion which has the form:

$$
\mathscr{E}_{\mathrm{ad}}=A_{\mathrm{BAR}} W \text { with } W<0,
$$

where $W$ is the adhesion energy per unit area. Inserting the eqn (13) and (14) into the inequality $\mathscr{E}_{\text {be }}+\mathscr{E}_{\text {ad }}<0$, one obtains the lower bound:

$$
|W|>|W|_{\min } \equiv 2 \kappa m_{1}^{2}
$$

for the adhesive strength $|W|$. Thus, in order to overcome the bending energy, the adhesive strength $|W|$ must exceed the value $|W|_{\min }$. For lipid bilayers with $\kappa \simeq 10^{-19} \mathrm{~J}$, this threshold value is given by $|W|_{\min } \simeq 0.4 \mathrm{~mJ} \mathrm{~m}^{-2}$. The latter adhesive strength represents a substantial energy density since the tension of rupture of a lipid bilayer is only a few $\mathrm{mJ} \mathrm{m} \mathrm{m}^{-2}$.

Now, consider a membrane segment of area $A$ that adsorbs a certain number $N_{\mathrm{BAR}}$ of BAR domain proteins from the exterior compartment as in Fig. 3(c). The corresponding protein coverage is given by:

$$
\Gamma_{\mathrm{BAR}}=N_{\mathrm{BAR}} / A \approx \Gamma_{\max } C_{\mathrm{BAR}} / K_{\mathrm{d}},
$$

with $\Gamma_{\max } \simeq 1 / A_{\mathrm{BAR}}$, where $C_{\mathrm{BAR}}$ and $K_{\mathrm{d}}$ now denote the bulk concentration and the desorption equilibrium constant of the BAR domain proteins in close analogy to the adsorption of small molecules and polymers as discussed before. Such a membrane segment will acquire the spontaneous curvature:

$$
m=A_{\mathrm{BAR}} m_{1} \Gamma_{\mathrm{BAR}}=\frac{A_{\mathrm{BAR}}}{2 R_{\mathrm{BAR}}} \Gamma_{\mathrm{BAR}} \text { for } \Gamma_{\mathrm{BAR}}<\Gamma_{*},
$$

as obtained by superimposing the curvatures induced by the single BAR domain proteins. The parameter $\Gamma *$ in eqn (17) corresponds to the critical coverage, at which the adsorbed proteins are expected to undergo an isotropic-nematic transition. Indeed, the curvature superposition should be valid as long as the lipid/protein membrane remains in an isotropic fluid state, which implies that the adsorbed BAR domain proteins undergo rotational and translational diffusion within the membrane. Since these proteins have a relatively large aspect ratio of about 9, they will undergo an 
isotropic-nematic transition with increasing coverage. For hard rods with this aspect ratio, computer simulations ${ }^{29}$ lead to the estimate $\Gamma_{*} \simeq 4.8 /(14.4 \mathrm{~nm})^{2} \simeq 1 /\left(43 \mathrm{~nm}^{2}\right)$ for the critical coverage, which corresponds to about half the maximal coverage. For $\Gamma_{\mathrm{BAR}}>\Gamma_{*}$, the bound BAR domain proteins will become orientationally ordered and the spontaneous curvature will no longer be isotropic.

\subsection{Magnitude of spontaneous tension}

The available estimates for the spontaneous curvature $m$ are now used to assess the range of values that the spontaneous tension $\sigma=2 \mathrm{~km}^{2}$ can assume. One then finds that this tension can vary over a wide range from $2 \times 10^{-8} \mathrm{~mJ} \mathrm{~m}^{-2}$ up to $1 \mathrm{~mJ} \mathrm{~m}^{-2}$, see Table 1. All $\sigma$-values in this table were obtained by using the typical value $\kappa \simeq 10^{-19} \mathrm{~J}$ for the bending rigidity.

The first column of Table 1 refers to lipid bilayers exposed to a mixture of two sugar molecules, the monosaccharide glucose and the trisaccharide raffinose, see ref. 30. Analyzing the shape fluctuations or undulations of quasi-spherical vesicles, a method called flicker spectroscopy, the spontaneous curvature was found to be about $0.01 \mu^{-1}$ to $0.1 \mu \mathrm{m}^{-1}$, comparable to or somewhat larger than the mean curvature of the vesicle membranes. This spontaneous curvature agrees with theoretical estimates based on two depletion layers in front of the two membrane surfaces. ${ }^{12}$ These depletion layers differ in their thickness because of the different sizes of the two sugar molecules. The corresponding spontaneous tension $\sigma$ is rather small and varies between $2 \times 10^{-8} \mathrm{~mJ} \mathrm{~m}^{-2}$ and $2 \times 10^{-6} \mathrm{~mJ} \mathrm{~m}^{-2}$. In fact, the experimental method of flicker spectroscopy is limited to relatively small values of $m$ and $\sigma$ since the spontaneous tension $\sigma$ acts to suppress the bending undulations.

The second column in Table 1 corresponds to the adsorption of anchored polymers as discussed in subsection 3.2. As mentioned in this subsection, one system that was studied experimentally is provided by biotinylated DNA strands adsorbed to membrane-anchored avidin. ${ }^{21}$ In this case, the spontaneous curvature was estimated to lie between $0.1 \mu \mathrm{m}^{-1}$ and $1 \mu \mathrm{m}^{-1}$, which implies that $\sigma$ varies from $2 \times 10^{-6} \mathrm{~mJ} \mathrm{~m}^{-2}$ to $2 \times 10^{-4} \mathrm{~mJ} \mathrm{~m}^{-2}$.

The values in the third column of Table 1 have been obtained for vesicles that contain aqueous solutions of PEG and dextran molecules. ${ }^{11}$ This system represents a very instructive example, for which the spontaneous tension $\sigma$ can be directly measured as explained in section 6 below. As a result, one finds that $\sigma$ varies between $2 \times 10^{-3} \mathrm{~mJ} \mathrm{~m}^{-2}$ and $2 \times 10^{-2} \mathrm{~mJ} \mathrm{~m}^{-2}$, from which one estimates the spontaneous curvature to lie between $3 \mu \mathrm{m}^{-1}$ and $10 \mu \mathrm{m}^{-1}$.

The last column in Table 1 corresponds to BAR domain proteins as discussed in section 3.3 above. In this case, the spontaneous curvature $m$ depends on the protein coverage $\Gamma$, see eqn (17), and satisfies $m \leq m_{1}$ with $m_{1}=1 /\left(2 R_{\mathrm{BAR}}\right) \leqq 1 /(20 \mathrm{~nm})$ as in eqn (12). The latter $m$-value implies the rather large tension value $\sigma \simeq 1 \mathrm{~mJ} \mathrm{~m}^{-2}$, which is, in fact, only somewhat smaller than the tension of membrane rupture.

\section{Spontaneous tubulation of supported bilayer membranes}

The tubulation of supported bilayers has been observed in several recent experiments. ${ }^{7,10,31}$ In these experiments, amphiphatic, antimicrobial peptides were added

Table 1 Spontaneous (or preferred) curvatures $m$ in units of $\mu \mathrm{m}^{-1}$ and associated spontaneous tensions $\sigma$ in units of $2 \mathrm{~mJ} \mathrm{~m}^{-2}$ for four different membrane systems with bending rigidity $\kappa \simeq 10^{-19} \mathrm{~J}$

\begin{tabular}{lllll}
\hline & Sugar solutions $^{30}$ & DNA strands & PEG/dextran solutions ${ }^{11}$ & BAR domains ${ }^{3,22}$ \\
\hline$m\left[\mu \mathrm{m}^{-1}\right]$ & $0.01-0.1$ & $0.1-1$ & $3-10$ & $10-50$ \\
$\sigma\left[2 \mathrm{~mJ} \mathrm{~m}^{-2}\right]$ & $10^{-8}-10^{-6}$ & $10^{-6}-10^{-4}$ & $10^{-3}-10^{-2}$ & $10^{-2}-0.5$ \\
\hline
\end{tabular}


to the exterior solution, which then adsorbed onto the supported membranes. Rather long tubules were observed that grew relatively slowly from these membranes. For example, when cyclic hexapeptide C-RW molecules were adsorbed on negatively charged POPG membranes, a process studied in ref. 10, the length of some tubules exceeded $100 \mu \mathrm{m}$ after one hour with a diameter between 1 and $4 \mu \mathrm{m}$. In the present section, I will argue that this tubulation process is driven by the spontaneous tension generated in the bilayer membranes by the adsorption-induced spontaneous curvature. The latter curvature can be directly estimated from the diameter of the tubules.

\subsection{Planar membranes adhering to solid surfaces}

In order to understand the main physical mechanism underlying the spontaneous tubulation of membranes and vesicles, we will first consider a large supported membrane that adheres to a solid surface. The membrane has the area $A$ and its shape energy is given by:

$$
\mathscr{E}=\mathscr{E}_{\mathrm{be}}+W A,
$$

with the bending energy as in eqn (1) and the adhesion energy density $W<0$.

If the membrane has no spontaneous curvature, the membrane will be planar with mean curvature $M=m=0$. Now, assume that some molecules are added to the exterior compartment and that these molecules adsorb onto the supported membrane, thereby generating a spontaneous mean curvature $m>0$ in this membrane. Because of this curvature, the flat state will acquire the bending energy $\mathscr{E}_{\mathrm{be}}=2 \mathrm{~km}^{2} A=\sigma A$ and the shape energy assumes the form:

$$
\mathscr{E}=2 \kappa m^{2} A+W A=(\sigma-|W|) A .
$$

This shape energy is positive if the spontaneous tension $\sigma$ exceeds the adhesive strength $|W|$, i.e., if

$$
\sigma>\sigma_{*} \equiv|W|
$$

which defines the tension threshold $\sigma *$. Therefore, for sufficiently large spontaneous tension exceeding this threshold, the supported membrane prefers to unbind from the solid surface. However, the planar membrane also needs to undergo a shape transformation during its unbinding process.

Indeed, let us first assume that a membrane segment of area $\Delta A$ remains planar as it unbinds. Such a process is energetically unfavorable since the unbound membrane segment has the shape energy $\sigma \Delta A$, which is larger than the shape energy $(\sigma-|W|)$ $\Delta A$ of the bound segment. Thus, as the membrane segment unbinds, it needs to deform in such a way that its mean curvature $M$ becomes close to the spontaneous curvature $m$. Two possible shape transformations come to mind: budding as shown in Fig. 4(a,b) and tubulation as in Fig. 4(c). It turns out that, for supported membranes with a uniform composition, bud formation is energetically unfavorable, whereas tubulation can be used to relax the spontaneous tension. This distinction will now be derived by considering three pathways: budding of an intramembrane domain, budding of a uniform membrane, and tubulation of a uniform membrane.

\subsection{Domain-induced budding of supported membrane}

First, assume that the adsorption of the molecules leads to phase separation into fluid $\mathrm{a}$ and $\mathrm{b}$ phases and that this process proceeds via the formation of $\mathrm{b}$-domains surrounded by an a-phase. Such domains were first considered theoretically and predicted to undergo budding transformations. ${ }^{32,33}$ Both the formation of these 


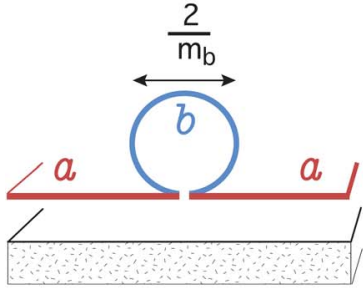

(a)

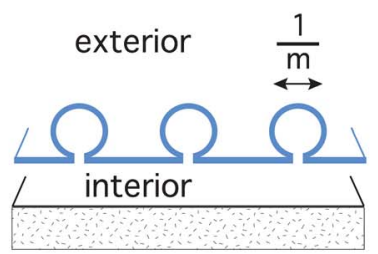

(b)

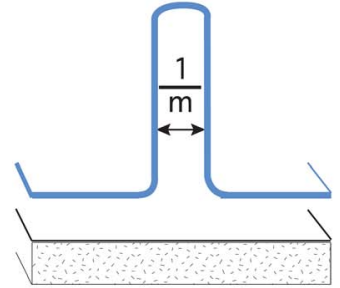

(c)

Fig. 4 Supported bilayer membranes that acquire a spontaneous curvature $m>0$ via adsorption form the exterior compartment may undergo budding or tubulation in order to adapt their shape to this curvature: (a) if the supported membrane undergoes phase separation into intramembrane domains of the b-phase (blue), that acquire a spontaneous curvature $m_{\mathrm{b}}$, which is large compared to the one in the mother membrane consisting of a-phase (red), the b-domains form spherical buds with a diameter of the order of $2 / m$; (b) if the whole planar membrane acquires the uniform spontaneous curvature $m>0$, it can again form spherical buds but these buds now have a smaller diameter of the order of $1 / \mathrm{m}$. As a consequence, this process is energetically unfavorable and the corresponding energy cost grows linearly with the number of buds; and (c) a much better alternative for the uniform membrane is to unbind from the substrate by forming a nanotube with diameter $1 / m$, which has vanishing bending energy apart from the contributions of the two tube ends.

domains and the associated budding process have been confirmed experimentally by fluorescence microscopy of giant vesicles. ${ }^{34-41}$

If the b-domains have a spontaneous curvature $m_{\mathrm{b}}$, which is large compared to the spontaneous curvature $m_{\mathrm{a}}$ of the mother membrane consisting of a-phase, the b-domains will undergo a budding transformation ${ }^{32}$ and will form spherical buds with radius $R_{\text {bud }}=1 / m_{\mathrm{b}}$ and bud diameter $2 / m_{\mathrm{b}}$ as shown in Fig. 4(a). The existence of such buds follows from the generalized neck condition: ${ }^{33}$

$$
\left(M_{\mathrm{a}}-m_{\mathrm{a}}\right)+\left(M_{\mathrm{b}}-m_{\mathrm{b}}\right)=\lambda /(2 \kappa)
$$

for the mean curvatures $M_{\mathrm{a}}$ and $M_{\mathrm{b}}$ of the two domains close to their joint neck, where $\lambda$ represents the line tension of the domain boundary. If the planar a-membrane with $M_{\mathrm{a}}=0$ has no spontaneous curvature, i.e., $m_{\mathrm{a}}=0$, the neck condition (21) simplifies and becomes:

$$
M_{\mathrm{b}}=m_{\mathrm{b}}+\lambda /(2 \kappa)=1 / R_{\mathrm{b}} .
$$

A planar b-domain with area $\Delta A_{\mathrm{b}}=4 \pi R_{\mathrm{b}}^{2}$ has the shape energy

$$
\mathscr{E}_{\mathrm{pl}}=4 \pi R_{\mathrm{b}}^{2}\left(\sigma_{\mathrm{b}}-|W|\right)+2 \pi R_{\mathrm{b}} \lambda \text { with } \sigma_{\mathrm{b}} \equiv 2 \kappa m_{\mathrm{b}}^{2}
$$

As this planar b-domain is transformed into a spherical bud with curvature radius $R_{\mathrm{b}}$, its shape energy becomes:

$$
\mathscr{E}_{\text {bud }}=4 \pi R_{\mathrm{b}}^{2} 2 \kappa\left(M_{\mathrm{b}}-m_{\mathrm{b}}\right)^{2}=4 \pi R_{\mathrm{b}}^{2} \lambda^{2} /(2 \kappa) .
$$

The budding transformation of the domain then leads to the energy change: $\S$

$$
\Delta \mathscr{E}_{1} \equiv \mathscr{E}_{\mathrm{bud}}-\mathscr{E}_{\mathrm{pl}}=4 \pi R_{\mathrm{b}}^{2}\left(\sigma_{\mathrm{b}^{*}}-\sigma_{\mathrm{b}}\right)
$$

with the tension threshold

\footnotetext{
$\S$ The b-bud is connected to the a-membrane by a small neck with constant mean curvature and zero bending energy.
} 


$$
\sigma_{\mathrm{b} *} \equiv|W|-\frac{1}{2} \lambda m_{\mathrm{b}}\left[1-\lambda /\left(2 \kappa m_{\mathrm{b}}\right)\right]
$$

Thus, the planar b-domain and the spherical b-bud have the same shape energy for $\sigma_{\mathrm{b}}=\sigma_{\mathrm{b}^{*}}$, and the transformation from the planar b-domain to the spherical bud is energetically favorable if

$$
\Delta \mathscr{E}_{1}<0 \text { or } \sigma_{\mathrm{b}}>\sigma_{\mathrm{b}^{*}}
$$

Thus, in the latter case, the bound b-segment can get rid of the adsorption-induced tension $\sigma$ by forming spherical buds.

Compared to the uniform membrane, see eqn (20), the tension threshold $\sigma_{\mathrm{b}^{*}}$ of the b-domain as given by eqn (26) involves an additional term proportional to the line tension $\lambda$. This term is negative implying:

$$
\sigma_{\mathrm{b}^{*}} \leq|W| \text { for } m_{\mathrm{b}} \geq \lambda /(2 \kappa) \text {. }
$$

For bending rigidity $\kappa \simeq 10^{-19} \mathrm{~J}$ and line tension $\lambda \simeq 10^{-13} \mathrm{~J} \mathrm{~m}^{-1}$, the ratio $\lambda /(2 \kappa) \simeq 1 /(2 \mu \mathrm{m})$.

In general, domain-induced budding has to overcome an energy barrier. This energy barrier is large for small domains and decreases with increasing domain size $L$ until it vanishes at a certain critical size $L_{\mathrm{o}}$, which behaves as ${ }^{32}$

$$
L_{\mathrm{o}} \approx 2 / m \text { for small } \lambda /\left(\kappa m_{\mathrm{b}}\right) \text {. }
$$

In the same limit, the energy barrier at the transition point with $\sigma_{\mathrm{b}}=\sigma_{\mathrm{b}^{*}}$ vanishes as

$$
\Delta E_{\mathrm{ba}} \approx q \kappa\left(\lambda / \kappa m_{\mathrm{b}}\right)^{4 / 3},
$$

with the dimensionless coefficient $q \simeq 2.2$.

\subsection{Budding of supported membrane with uniform composition}

Next, assume that the whole supported membrane acquires the adsorption-induced spontaneous curvature $m$ and that the corresponding spontaneous tension $\sigma=2 \mathrm{~km}^{2}$ satisfies $\sigma>|W|$. In this case, the membrane may again try to unbind by the formation of spherical buds but these buds are more strongly curved with radius $R_{\text {bud }}=1 /(2 m)$ and bud diameter $1 / m$, see Fig. 4(b). This increased curvature follows from the neck condition for uniform membranes as in eqn (11), which now becomes:

$$
m=\frac{1}{2}\left(M_{\mathrm{pl}}+M_{\mathrm{bud}}\right)=\frac{1}{2} M_{\mathrm{bud}}=1 /\left(2 R_{\mathrm{bud}}\right) .
$$

The area of such a bud is equal to:

$$
A_{\mathrm{bud}}=4 \pi R_{\mathrm{bud}}^{2}=\pi / m^{2} .
$$

A planar membrane segment with area $\Delta A=A_{\text {bud }}$ has the shape energy:

$$
\mathscr{E}_{\mathrm{pl}}=(\sigma-|W|) A_{\text {bud }}=2 \kappa \pi-|W| A_{\text {bud }}
$$

When this segment is transformed into a spherical bud with mean curvature $M_{\text {bud }}=2 m$, its shape energy becomes:

$$
\mathscr{E}_{\text {bud }}=A_{\text {bud }} 2 \kappa\left(M_{\text {bud }}-m\right)^{2}=2 \pi \kappa .
$$


Therefore, the budding transformation now leads to the energy change:

$$
\Delta \mathscr{E}_{2}=\mathscr{E}_{\text {bud }}-\mathscr{E}_{\mathrm{pl}}=|W| A_{\text {bud }}=\pi|W| / m^{2},
$$

which is positive and thus represents an energy cost as long as the solid surface is adhesive with $W<0$. Thus, the formation of $N_{\text {bud }}$ such buds leads to the energy $\operatorname{cost} N_{\text {bud }} \Delta \mathscr{E}_{2}=N_{\text {bud }} \pi|W| / m^{2}$, which grows linearly with $N_{\text {bud. }}$ Therefore, the formation of buds from a supported membrane with uniform composition and, thus, uniform spontaneous curvature $m$ does not provide a downhill pathway for the unbinding of the supported membrane.

\subsection{Tubulation of supported membrane with uniform composition}

An energetically much more favorable pathway for the unbinding of a uniform membrane with spontaneous curvature $m$ consists in the formation of cylindrical tubules as shown in Fig. 4(c). These tubules have radius $R_{\mathrm{cy}}=1 / 2 \mathrm{~m}$, which is equal to the radius $R_{\mathrm{sp}}=1 / 2 \mathrm{~m}$ of the spherical buds in Fig. 4(b), but their mean curvature $M=1 / 2 R_{\mathrm{cy}}=m$ leads to a vanishing bending energy in contrast to the buds, which have mean curvature $M=1 / R_{\mathrm{sp}}=2 \mathrm{~m}$. As before, a planar membrane segment with area $\Delta A$ has the shape energy:

$$
\mathscr{E}_{\mathrm{pl}}=(\sigma-|W|) \Delta A
$$

If this segment is now transformed into a cylindrical tubule with mean curvature $M=m$ and radius $1 / 2 m$ as in Fig. 4(c), the bending energy vanishes along the main body of the tubule and its shape energy is equal to the bending energy contributions from the two ends of the tube, i.e.,

$$
\mathscr{E}_{\text {tub }}=\mathscr{E}_{\text {end }},
$$

which becomes independent of the tube area $\Delta A$ for large $\Delta A$. The transformation of the planar segment to the tubule thus leads to the energy change:

$$
\Delta \mathscr{E}_{3}=\mathscr{E}_{\text {tub }}-\mathscr{E}_{\mathrm{pl}}=\mathscr{E}_{\text {end }}-(\sigma-|W|) \Delta A,
$$

which behaves as

$$
\Delta \mathscr{E}_{3} \approx-(\sigma-|W|) \Delta A \text { for large } \Delta A
$$

corresponding to long tubes. Therefore, if the supported membrane acquires a uniform spontaneous curvature $m$ that leads to a spontaneous tension $\sigma>|W|$, the membrane will unbind from the solid surface via the formation of tubules. Vice versa, if we transform a long tubule with area $\Delta A$ back into a planar membrane segment, we need to perform the (reversible) work $(\sigma-|W|) \Delta A$.

\subsection{Stability of cylindrical tubules and osmotic conditions}

One might expect that a cylindrical tubule as in Fig. 4(c) undergoes another shape transformation, in analogy to the classical Rayleigh-Plateau instability of liquid cylinders, and then forms a string of small spheres. A liquid cylinder of constant volume is unstable with respect to such a transformation because a string of spherical droplets has a smaller interfacial area and, thus, a smaller interfacial free energy. This reduction in free energy is, however, not available to the cylindrical tubule since any shape transformation of the tube must conserve its membrane area.

Thus, let us compare a cylindrical tubule of radius $R_{\mathrm{cy}}=1 / 2 \mathrm{~m}$ with a necklace-like tubule consisting of a string of small spheres with radius $R_{\mathrm{sp}}=1 / \mathrm{m}$. Ignoring again the contributions from the tube ends, both shapes have the same mean curvature 
$M=m$ and, thus, vanishing bending energy, However, these two shapes differ in their volume which implies that their energy difference will depend on the osmotic pressures. A cylindrical tubule of radius $R_{\mathrm{cy}}=1 / 2 \mathrm{~m}$ and length $L$ has the volume:

$$
V_{\mathrm{cy}}=\pi R_{\mathrm{cy}}^{2} L=\pi L /\left(4 m^{2}\right) .
$$

If we transform this tubule into a string of small spheres with radius $R_{\mathrm{sp}}=1 / \mathrm{m}$ for fixed membrane area, we obtain:

$$
N_{\mathrm{ss}}=m L / 4
$$

such spheres. These spheres have the volume:

$$
V_{\mathrm{ss}}=N_{\mathrm{ss}} \frac{4 \pi}{3} R_{\mathrm{sp}}^{3}=\pi L /\left(3 m^{2}\right)>V_{\mathrm{cy}} .
$$

Thus, during the transformation of a cylindrical into a necklace-like tubule, the tubule volume is increased by:

$$
\Delta V_{\mathrm{tub}}=V_{\mathrm{ss}}-V_{\mathrm{cy}}=\pi L /\left(12 m^{2}\right),
$$

proportional to the tube length $L$, and the tubule must perform the mechanical work:

$$
\Delta \mathscr{E}_{4}=\Delta V_{\text {tub }}\left(P_{\text {tub,ex }}-P_{\text {tub,in }}\right)
$$

where $P_{\text {tub,ex }}$ and $P_{\text {tub,in }}$ represent the osmotic pressure within the exterior compartment and the tubule, respectively. The energy change $\Delta \mathscr{E}_{4}$ is positive for $P_{\text {tub,ex }}>$ $P_{\text {tub,in }}$ and negative for $P_{\text {tub,ex }}<P_{\text {tub,in. }}$. Thus, the cylindrical tubule will be stable if the exterior osmotic pressure exceeds the osmotic pressure within the tubule. This conclusion agrees with the results of linear stability analysis as performed in ref. 42-44.

When the adsorbing particles are added to the exterior compartment of a supported bilayer, these particles increase the osmotic pressure within this compartment. Thus, the osmotic pressure difference $P_{\text {tub,ex }}-P_{\text {tub,in }}$ should be positive after the addition of the particles. However, as time goes on, this osmotic pressure difference can relax via defects in the supported bilayer. For $P_{\text {tub,ex }}=P_{\text {tub,in, no }}$ work is necessary to transform the cylindrical tubule into the necklace-like tubule. Since both tubule morphology have the same bending energy apart from the contributions at the tube ends, the latter contributions are now essential to determine, which morphology of the tubule is energetically more favorable. The experimental observations in ref. 10 indicate that the necklace-like morphology is energetically more favorable for the distal tube end not connected to the supported bilayer.

\subsection{Value of spontaneous curvature}

The previous considerations show that the supported membrane can unbind from the substrate surface by forming long tubules that protrude into the exterior compartment provided the spontaneous tension $\sigma$ generated by the adsorptioninduced spontaneous curvature $m$ exceeds the tension threshold $\sigma_{*}$ of the supporting substrate, which is equal to the adhesive strength $=|W|$ of this substrate. Since $\sigma=$ $2 \mathrm{Km}^{2}$, this condition is equivalent to the inequalities:

$$
m>m_{*} \equiv(|W| / 2 \kappa)^{1 / 2} \text { and }|W|<|W|_{*} \equiv 2 \kappa m^{2}
$$

for the spontaneous curvature and the adhesive strength, respectively. During the adsorption process, the spontaneous curvature $m$ grows continuously until it exceeds 
the threshold value $m_{*}$. For $m>m_{*}$, the planar, supported membrane can reduce its shape energy by forming unsupported tubules with mean curvature $m>m *$. The initial shape transformation will encounter an energy barrier, which is expected to be of the order of the bending energy contribution $\mathscr{E}_{\text {end }}$ from the two tube ends, but could be strongly reduced by domain-induced budding.

The tubules of POPG membranes as observed in ref. 10 after the adsorption of cyclic hexapeptide C-RW had a diameter that varied between 1 and $4 \mu \mathrm{m}$. As shown in Fig. 4, the diameter of a cylindrical tubule is equal to the inverse spontaneous curvature. Therefore, in these experiments, the adsorption process induced a spontaneous curvature $m$ within the range

$$
1 /(4 \mu \mathrm{m}) \lesssim m \lesssim 1 / \mu \mathrm{m}
$$

Inserting the smaller $m$-value together with the putative bending rigidity value $\kappa \simeq$ $10^{-19} \mathrm{~J}$ into the relation (45), one obtains the bound

$$
|W|<|W|_{*} \simeq 10^{-5} \mathrm{~mJ} \mathrm{~m}^{-2}
$$

for the adhesive strength $|W|$ between the supported membrane and the supporting substrate surface. Thus, the theory described here predicts that the adhesive strength $|W|$ of the supporting substrate in ref. 10 is smaller than $10^{-5} \mathrm{~J} \mathrm{~m}^{-2}$, corresponding to relatively weak adhesion.

\subsection{Tubulation of supported bilayers on deformable substrates}

Recently, tubulation was also observed for lipid bilayers supported by deformable polydimethylsiloxane (PDMS) sheets with a thickness of about $100 \mu \mathrm{m} .{ }^{45}$ Initially, the PDMS sheets were planar and the supported bilayers were exposed to vesicles that adsorbed onto the bilayers. The sheets were then bent and stretched by applying a hydrostatic pressure. After this pressure was switched off, the sheets relaxed to their planar state and were, thus, compressed. This relatively fast compression induced the formation of many tubules that emanated from the supported bilayers. The excess membrane area for these tubules should arise from the adsorbed vesicles, after they have fused with the stretched bilayers. Since the volume of the tubules grows continuously during their formation process, they must be connected to water reservoirs, which are presumably provided by water layers between the supported bilayers and the PDMS sheets. If these water layers are relatively thin and the original defects in the supported bilayers have been closed by fusion with the adsorbed vesicles, the small available water volume could favor the formation of membrane tubes kinetically. Thus, it will be interesting to see if the tube formation persists when the compression speed of the PDMS sheets is strongly reduced. Tube formation during slow compression would imply that the tubules are again stabilized by spontaneous curvature.

\section{Spontaneous tubulation of giant vesicles}

\subsection{Giant vesicles with spherical membrane segments}

Giant vesicles with a radius of the order of $10 \mu \mathrm{m}$ can attain a large variety of shapes, which have been studied for a long time, both experimentally and theoretically. Here, I will focus on the shapes of visually tense vesicles that exhibit large spherical segments with a well-defined curvature radius $R_{\mathrm{sp}}$ that can be directly measured in the optical microscope. Some examples are shown in Fig. 5.

Spherical shapes are always found when the vesicles are slightly inflated by decreasing the osmotic pressure in the surrounding solution, spherical segments are observed for strong vesicle adhesion to solid or rigid substrate surfaces as well as in micropipette experiments, where their curvature radius is used in order to 
deduce the membrane tension from the applied aspiration pressure. ${ }^{48}$ Likewise, the shapes of vesicle membranes that enclose a phase-separated solution of PEG and dextran are provided by two spherical caps around the two coexisting liquid droplets.

\subsection{Shape energy of vesicles}

The vesicle shapes shown in Fig. 5 are determined by the osmotic conditions, by the elastic properties that determine the membrane's bending energy, and by the membrane tension. The osmotic pressures in the interior and exterior aqueous compartment will be denoted by $P_{\mathrm{sp}, \text { in }}$ and $P_{\mathrm{sp}, \mathrm{ex}}$, respectively, their pressure difference by:

$$
\Delta P \equiv P_{\mathrm{sp}, \mathrm{in}}-P_{\mathrm{sp}, \mathrm{ex}} .
$$

For isothermal processes as considered here, the total membrane area is constant. It is then convenient to incorporate the constraint of constant area by introducing the auxiliary tension $\Sigma$ that plays the role of a Lagrange multiplier. The shape energy of the vesicle membrane now has the form:

$$
\mathscr{E}=-\Delta P V+\Sigma A+\mathscr{E}_{\text {be }},
$$

with the bending energy $\mathscr{E}_{\text {be }}$ as in eqn (1).

As emphasized in section 2.3, the bending energy contains another term proportional to membrane area, which defines the spontaneous tension $\sigma=2 \mathrm{~km}^{2}$ as in eqn (5). When the latter tension is combined with the auxiliary tension $\Sigma$, one obtains the total membrane tension:

$$
\hat{\Sigma} \equiv \Sigma+\sigma=\Sigma+2 \kappa m^{2}
$$

(a)

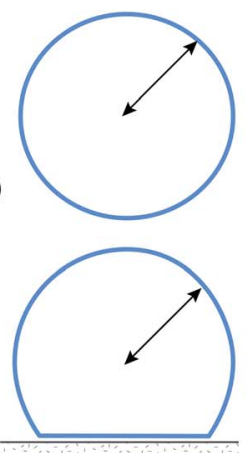

(b)

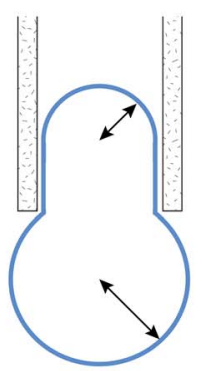

(c)

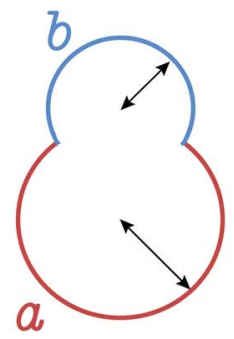

(d)

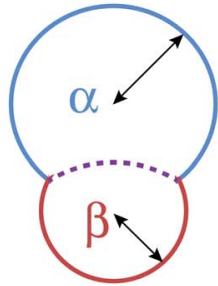

(e)

Fig. 5 Examples for tense vesicle membranes (blue or blue/red) with spherical segments as visible in the optical microscope: (a) vesicles attain a spherical shape after osmotic inflation; (b) vesicles that adhere strongly to a solid substrate (shaded) form spherical cap shapes; (c) vesicles that are aspirated into micropipettes (shaded) form two spherical membrane caps connected by a cylindrical one; $(\mathrm{d}, \mathrm{e})$ vesicle shapes consisting of two spherical caps that (d) are formed by two coexisting intramembrane domains, a (red) and b (blue), and (e) enclose two coexisting liquid droplets, $\alpha$ and $\beta$, which are separated by a liquid-liquid interface (purple broken line). The black double-arrows specify the various curvature radii $R_{\mathrm{sp}}$ of the spheres and spherical caps. The sharp membrane kinks at the boundaries of the spherical caps reflect the optical resolution and represent membrane segments that are smoothly bent on smaller length scales. ${ }^{46,47}$ It will become clear in the following that all of these vesicle shapes may also contain stable nanotubes that point towards the interior of the vesicles. 
For the membrane systems considered here, the large spherical segments may be regarded as area reservoirs for the tubules. The auxiliary tension $\Sigma$ then vanishes for large curvature radii $R_{\mathrm{sp}}$ of the spherical segments as will be shown below. Therefore, the giant vesicle systems considered here are, in fact, characterized by $|\Sigma| \ll \sigma$ and $\hat{\Sigma} \approx \sigma$.

\subsection{In-tubes and out-tubes connected to spherical membranes}

To proceed, let us now consider a vesicle as shown in Fig. 6(a,b) that has the shape of a large sphere with radius $R_{\mathrm{sp}}$ and a cylindrical tube with radius $R_{\mathrm{cy}}$ and length $L$. Alternatively, we may consider any of the spherical membrane segments depicted in Fig. 5 as will become clear in the next subsection. Likewise, as shown in Appendix B. $8, \uparrow$ the results obtained for a vesicle with one tubule also holds for a vesicle with several tubules as in Fig. 6(c).

The spherical vesicle in Fig. 6 is assumed to be so large that its radius $R_{\mathrm{sp}}$ can be directly measured by optical microscope. Thus, this radius will be treated as an observable parameter. In contrast, the tube radius $R_{\text {cy }}$ satisfies $R_{\text {cy }} \ll R_{\text {sp }}$ and will typically lie below the optical resolution. As shown in Fig. 6, it is important to distinguish two different cases, out-tubes and in-tubes as shown in Fig. 6(a) and (b,c), respectively.

Out-tubes and in-tubes differ in several important aspects. First, the out- and intubes are connected to different volume reservoirs: the out-tubes exchange aqueous solution with the interior vesicle compartment, which has a volume of the order of $R_{\mathrm{sp}}^{3}$, whereas the in-tubes exchange volume with the exterior compartment, which represents an effectively infinite volume reservoir. Second, the membranes of the out- and in-tubes experience different osmotic pressure differences as given by:

$$
\begin{aligned}
P_{\mathrm{cy}} \equiv P_{\mathrm{cy}, \mathrm{in}}-P_{\mathrm{cy}, \mathrm{ex}} & =+\Delta P \text { for out-tubes } \\
& =-\Delta P \text { for in-tubes. }
\end{aligned}
$$

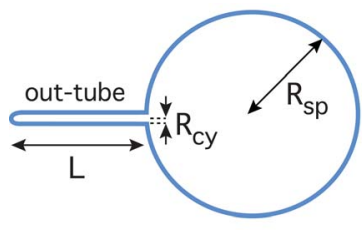

(a)

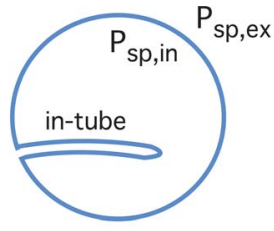

(b)

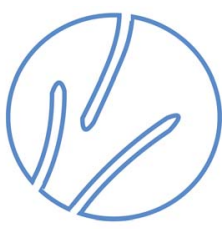

(c)

Fig. 6 Schematic cross-sections through spherical vesicles with radius $R_{\mathrm{sp}}$ connected to membrane tubes with radius $R_{\mathrm{cy}} \ll R_{\mathrm{sp}}$ and length $L$ : (a) out-tube protruding into the exterior compartment with positive mean curvature $M_{\mathrm{cy}}=1 / 2 R_{\mathrm{cy}}$; (b) in-tube protruding into the interior compartment of the vesicle with negative mean curvature $M_{\mathrm{cy}}=-1 / 2 R_{\mathrm{cy}}$; and (c) vesicle with three in-tubes. The schematic drawings are somewhat idealized because (i) the tube radii will usually be much smaller compared to the size of the spherical vesicle and (ii) the tubes undergo thermally-excited bending undulations and, thus, move in and out of the focal plane in the absence of external forces that pull at the tube ends. The osmotic pressures in the interior and exterior compartments are denoted by $P_{\mathrm{sp}, \text { in }}$ and $P_{\mathrm{ex}, \mathrm{sp}}$, respectively. For $\Delta P=P_{\mathrm{sp}, \text { in }}-$ $P_{\text {ex,sp }}>0$, the osmotic pressures act to expand the sphere and the out-tube but to compress the in-tube. The spherical vesicle has a sufficiently large radius $R_{\mathrm{sp}}$ that can be directly measured by optical microscopy, whereas the tube radius $R_{\mathrm{cy}}$ is typically below optical resolution. The stress balance conditions for these sphere/tube systems also apply when the sphere is replaced by spherical membrane segments as in Fig. 5. 
Thus, the membrane of an out-tube is subject to the same pressure difference as the membrane of the large spherical segment whereas an in-tube feels the opposite pressure difference. Third, because of the sign convention introduced in Fig. 2, an out-tube has positive mean curvature $M_{\mathrm{cy}}=+1\left(2 R_{\mathrm{cy}}\right)$ whereas an in-tube has negative mean curvature $M_{\mathrm{cy}}=-1\left(2 R_{\mathrm{cy}}\right)$.

\subsection{Stress balance for spherical membranes and cylindrical tubes}

In mechanical equilibrium, the spherical segment attains a certain curvature radius $R_{\mathrm{sp}}$ and a certain mean curvature $M_{\mathrm{sp}}=1 / R_{\mathrm{sp}}$ that is characterized by vanishing stress in the radial direction perpendicular to the membrane. As shown in Appendix B. $1, \dagger$ one then obtains the radial stress balance relation:

$$
\Delta P=2 \hat{\Sigma} M_{\mathrm{sp}}-4 \kappa m M_{\mathrm{sp}}^{2}=2 \Sigma M_{\mathrm{sp}}+4 \kappa m^{2} M_{\mathrm{sp}}\left(1-M_{\mathrm{sp}} / m\right),
$$

with the total membrane tension $\hat{\Sigma}=\Sigma+2 \kappa m^{2}$ as in (50). A spherical membrane segment that satisfies this radial stress balance condition will be regarded as 'stress-free'. $\uparrow$

A cylindrical tubule is characterized by its radius $R_{\text {cy }}$ and its length $L$, see Fig. 6 . Mechanical equilibrium of such a tubule implies two stress balance relations, one for the radial direction perpendicular to the cylindrical membrane and one for the axial direction parallel to cylindrical axis. These two relations can be solved for the total tension $\hat{\Sigma}$ and the osmotic pressure difference $\Delta P$. In the absence of external forces, one then obtains the total tension:

$$
\hat{\Sigma}=8 \kappa m M_{\text {cy }}-6 \kappa M_{\text {cy }}^{2},
$$

the auxiliary tension

$$
\Sigma=\hat{\Sigma}-\sigma=-6 \kappa\left(M_{\mathrm{cy}}-m\right)\left(M_{\mathrm{cy}}-\frac{1}{3} m\right)
$$

and the osmotic pressure difference

$$
\Delta P=16 \kappa M_{\mathrm{cy}}^{2}\left(m-M_{\mathrm{cy}}\right),
$$

see Appendix B.3.† A cylindrical tube that satisfies these relations will be considered as 'stress-free'.

\subsection{In- and out-tubes connected to large vesicles}

So far, the spherical vesicle membrane and the cylindrical tubules have been considered separately. Now, we take into account that these two segments belong to the same membrane, which implies that the stress balance relations (53) and (55) for the tube must be fulfilled together with the stress balance relation (52) for the spherical membrane segment. ${ }^{49}$ One can then eliminate both the pressure difference $\Delta P$ and the auxiliary tension $\Sigma$ and obtain a functional relationship between the mean curvature $M_{\mathrm{cy}}$ of the cylindrical nanotube and the mean curvature $M_{\mathrm{sp}}$ of the spherical segment as well as the elastic membrane parameters $\kappa$ and $m$. As shown in Appendix B.6, $\uparrow$ this relation has the simple form

$$
M_{\mathrm{cy}} \approx m-\frac{1}{4} M_{\mathrm{sp}}\left[1-\frac{1}{4}\left(\frac{M_{\mathrm{sp}}}{m}\right)^{2}\right] \text { for small } M_{\mathrm{sp}} /|m|=2 R_{\mathrm{cy}} / R_{\mathrm{sp}},
$$

व The intuitive term 'stress-free' is used here and below for all shapes that correspond to extrema of the shape energy (49). If such an extremum represents a minimum, the stress-free shape is stable; if it represents a saddle point or maximum, the stress-free shape is unstable. 
with $m<0$ for in-tubes and $m>0$ for out-tubes. Since $M_{\mathrm{sp}}=1 / R_{\mathrm{sp}}>0$, the correction term is always negative and makes the mean curvature $M_{\mathrm{cy}}$ less positive for out-tubes but more negative for in-tubes. It follows from (56) that the tube radius is given by:

$$
R_{\mathrm{cy}}=\frac{1}{2 M_{\mathrm{cy}}} \approx \frac{1}{2 m}+\frac{1}{8 m^{2} R_{\mathrm{sp}}} \text { for out-tubes }
$$

and by

$$
R_{\mathrm{cy}}=-\frac{1}{2 M_{\mathrm{cy}}} \approx-\frac{1}{2 m}-\frac{1}{8 m^{2} R_{\mathrm{sp}}} \text { for in-tubes. }
$$

Therefore, as the curvature radius $R_{\mathrm{sp}}$ of the large sphere or spherical cap decreases, the radius $R_{\text {cy }}$ of a stress-free out-tube increases whereas the radius $R_{\text {cy }}$ of a stress-free in-tube decreases.

As shown in Appendix B.8, $\dagger$ it is straightforward to generalize these considerations to several tubes connected to the same spherical membrane segment. One then obtains the following two simple rules: (i) a spherical membrane segment cannot be connected, at the same time, to both stress-free out-tubes and stressfree in-tubes; and (ii) when connected to the same spherical segment, all stress-free out- and in-tubes have the same radius as given by (57) and (58), respectively, provided the membrane has uniform elastic properties. $\|$

\subsection{Stability of membrane tubes}

The local stability of membrane tubes has been theoretically studied in ref. 42-44. In the absence of external pulling forces, the results of these studies imply that the stability of these tubes are primarily determined by the osmotic pressure difference $P_{\text {cy }}=P_{\text {cy,in }}-P_{\text {cy,ex }}$ across the tube membranes. Thus, in order to determine the stability of the in- and out-tubes, we need to determine the pressure difference $\Delta P$ for the two types of tubes. This pressure difference is given by:

$$
\Delta P=16 \kappa M_{\mathrm{cy}}^{2}\left(m-M_{\mathrm{cy}}\right) \approx 4 \kappa m^{2} M_{\mathrm{sp}}-2 \kappa m M_{\mathrm{sp}}^{2}
$$

as follows from a combination of the eqn (55) and (56). In addition, since the leading term on the right hand side of (59) is positive irrespective of the sign of the spontaneous curvature $m$, the pressure difference $\Delta P$ becomes:

$$
\Delta P \approx 4 \kappa m^{2} M_{\mathrm{sp}} \approx 4 \kappa M_{\mathrm{cy}}^{2} M_{\mathrm{sp}}=\kappa /\left(R_{\mathrm{cy}}^{2} R_{\mathrm{sp}}\right)>0 \text { for }|m| \gg M_{\mathrm{sp}},
$$

a relation that applies to both stress-free out- and stress-free in-tubes.

At the same time, the pressure difference $P_{\mathrm{cy}}=P_{\mathrm{cy}, \text { in }}-P_{\mathrm{cy}, \mathrm{ex}}$ across the tube membranes is equal to $+\Delta P$ for out-tubes and to $-\Delta P$ for in-tubes as in (51). Therefore, the out-tubes experience a positive pressure difference $P_{\text {cy }}$ whereas the in-tubes feel a negative one. As a consequence, the out-tubes are unstable but the in-tubes are stable even in the absence of external forces that pull on these tubes, as shown more explicitly in Appendices B.10 and B.11. $\dagger$ The results of this linear stability analysis agree with the arguments presented in subsection 4.5 , where the volume of a cylindrical tube was shown to be smaller than the volume of a necklace-like tube bounded by the same membrane area.

\footnotetext{
|| It is straightforward to extend this approach to vesicles, for which the spherical membrane segment and the tubules consist of different intramembrane phases and, thus, have different elastic properties.
} 


\subsection{Dominance of spontaneous tension}

The total tension $\hat{\Sigma}=\Sigma+\sigma$ of a stress-free and stable in-tube is given by eqn (53), which depends on the bending rigidity $\kappa$, the spontaneous curvature $m<0$, and the tube's mean curvature $M_{\mathrm{cy}}<0$. Inserting expression (56) for $M_{\mathrm{cy}}$ into this relation, the total tension becomes:

$$
\hat{\Sigma} \approx 2 \kappa m^{2}+\kappa m M_{\mathrm{sp}}=\sigma+\kappa m M_{\mathrm{sp}} .
$$

It then follows that the auxiliary tension $\Sigma=\hat{\Sigma}-\sigma$ behaves as:

$$
\Sigma \approx \kappa m M_{\mathrm{sp}}=\frac{M_{\mathrm{sp}}}{2 m} \sigma
$$

or

$$
\Sigma \approx \frac{M_{\mathrm{sp}}}{2 M_{\mathrm{cy}}} \sigma \approx-\frac{R_{\mathrm{cy}}}{R_{\mathrm{sp}}} \sigma .
$$

Thus, in the limit of small $R_{\mathrm{cy}} / R_{\mathrm{sp}}$ corresponding to large spherical segments or thin tubes, the total tension $\hat{\Sigma}$ approaches the spontaneous tension $\sigma$, whereas the auxiliary tension $\Sigma \approx \kappa m M_{\mathrm{sp}}$ vanishes. Since the spontaneous curvature $m$ is negative for in-tubes, the auxiliary tension $\Sigma$ is negative, as well and its absolute value is smaller than the spontaneous tension $\sigma$ by a factor $R_{\mathrm{cy}} / R_{\mathrm{sp}} \ll 1$. Therefore, the total tension $\hat{\Sigma}$ of stable in-tubes protruding into the interior of large vesicles is dominated by the spontaneous tension $\sigma$.

The dominance of the spontaneous tension has also important consequences for the osmotic pressure difference $\Delta P$ as given by expression (52). First, this expression assumes the simpler form:

$$
\Delta P \approx 2 \Sigma M_{\mathrm{sp}}+4 \kappa m^{2} M_{\mathrm{sp}}=2 \hat{\Sigma} M_{\mathrm{sp}} \text { for }|m| \gg M_{\mathrm{sp}},
$$

which is a Laplace-like equation that is linear in $M_{\mathrm{sp}}$, compare Appendix B.2. $\dagger$ Inspection of (52) shows that the omitted nonlinear term is smaller by a factor $M_{\mathrm{sp}} /|m|$. Furthermore, because the auxiliary tension $\Sigma \approx\left(M_{\mathrm{sp}} / m\right) \sigma$ as in (62), the term proportional to $\Sigma$ is of the same order as the nonlinear one, and the osmotic pressure difference assumes the simple form:

$$
\Delta P=2 \sigma M_{\mathrm{sp}}\left[1+O\left(M_{\mathrm{sp}} /|m|\right)\right] \text { for }|m| \gg M_{\mathrm{sp}} .
$$

Thus, the presence of stable in-tubes with $\left|M_{\text {cy }}\right| \approx|m| \gg M_{\text {sp }}$ implies that the osmotic pressure difference $\Delta P$ is primarily balanced by the spontaneous tension $\sigma$. In general, the stress balance as described by (65) will always apply if the spontaneous curvature $m$ of the membrane is sufficiently large with $|m| \gg M_{\mathrm{sp}}$. Thus, the stress balance (65) could apply to all shapes depicted in Fig. 5 but this is difficult to decide unless one can detect the presence of nanotubes.

\section{Membrane tubulation in aqueous two-phase systems}

As shown in the previous section, the total membrane tension $\hat{\Sigma}=\Sigma+\sigma$ is governed by the spontaneous tension $\sigma=2 \mathrm{~km}^{2}$ for large spherical membrane segments connected to stable nanotubes. Therefore, if we could measure the total tension $\hat{\Sigma} \approx \sigma$, we could deduce the spontaneous (or preferred) curvature $m$ from this measurement. Such a deduction has been recently achieved for vesicles in contact with aqueous solutions of dextran and polyethylene glycol (PEG). ${ }^{11,50}$

When the polymer concentrations exceed a few weight percent, aqueous PEG/ dextran solutions undergo phase separation, and the lipid membranes are exposed 
to different aqueous phases. The phase separation process leads to partial or complete wetting of the membranes by the aqueous phases ${ }^{51}$ and induces shape transformations of the vesicles such as droplet-induced budding, ${ }^{50,52}$ which can be directly observed in the optical microscope. In addition, the phase separation process within the interior vesicle compartment also induces stable nanotubes that have a diameter below the resolution of conventional optical microscopy but can be observed by fluorescence microscopy. ${ }^{11}$

Phase separation within the vesicles can be induced by temperature changes ${ }^{53}$ or by osmotic deflation, ${ }^{51}$ i.e., by adding osmotically active particles to the exterior solution. The latter method has the advantage that the membrane area is not changed during the isothermal process. The resulting osmotic unbalance leads to water permeation through the vesicle membranes and to a reduced volume of the vesicles. Since the polymers cannot permeate the membrane, the polymer concentration is increased, and the aqueous solution inside the vesicles undergoes phase separation into the PEG-rich $\alpha$ phase and the dextran-rich $\beta$ phase. The theoretical considerations described in this section are, in fact, quite general and apply to vesicles in any aqueous solution that can undergo phase separation into two aqueous phases.

The phase separation process within a single vesicle proceeds via several stages that can be observed in the optical microscope. ${ }^{54}$ First, many small droplets are formed within the vesicle. These droplets then grow and coalesce until the interior compartment of the vesicle contains, apart from the nanotubes, only two large droplets, one $\alpha$ and one $\beta$ droplet. As a result, when viewed with conventional optical microscopy, a single vesicle typically attains a state as shown schematically in Fig. 5(e) and Fig. 7(a). In such a state, both liquid phases $\alpha$ and $\beta$ are in contact with the vesicle membrane corresponding to partial wetting of this membrane.

These vesicles have been addressed in several previous studies, both experimentally and theoretically. ${ }^{11,47,50,51}$ In addition, the experimental aspects of these systems have been recently reviewed in ref. 55. Therefore, I will focus here on two aspects that have not been pointed out in previous publications but are important from a conceptual point of view: (i) The effective contact angles as observed in the optical microscope are primarily determined by the spontaneous tensions and, thus, by the spontaneous curvatures of the different membrane segments; and (ii) The relation between the effective contact angles and the intrinsic one that was derived in ref. 47 under the simplifying assumption of vanishing spontaneous curvatures is, in fact, valid for arbitrary spontaneous curvatures.

\subsection{Vesicle geometry and total membrane tensions}

After the phase separation inside the vesicle has been completed, the vesicle membrane typically assumes the shape as shown in Fig. 7(a): the membrane encloses two liquid droplets consisting of the $\alpha$ and $\beta$ phase, respectively, which are separated by a normal liquid-liquid interface with interfacial tension $\Sigma_{\alpha \beta}$. This shape has several remarkable features.

First, the membrane consists of two membrane segments that form two spherical caps around the two droplets. One membrane segment separates the $\alpha$ droplet from the exterior phase $\gamma$, the other segment separates the $\beta$ droplet from $\gamma$. The two membrane segments will, in general, have different bending rigidities $\kappa_{\alpha \gamma}$ and $\kappa_{\beta \gamma}$ as well as different spontaneous curvatures $m_{\alpha \gamma}$ and $m_{\beta \gamma}$. The total tension of the $\alpha \gamma$ membrane is then given by:

$$
\hat{\Sigma}_{\alpha \gamma}=\Sigma+\sigma_{\alpha \gamma}=\Sigma+2 \kappa_{\alpha \gamma} m_{\alpha \gamma}^{2}
$$

and the total tension of the $\beta \gamma$ membrane by

$$
\hat{\Sigma}_{\beta \gamma}=\Sigma+\sigma_{\beta \gamma}=\Sigma+2 \kappa_{\beta \gamma} m_{\beta \gamma}^{2} .
$$




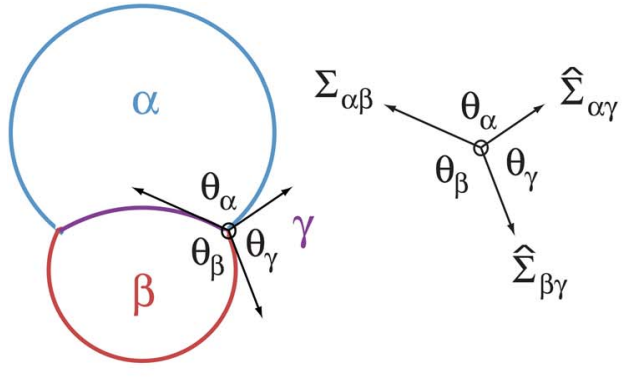

(a) (b)

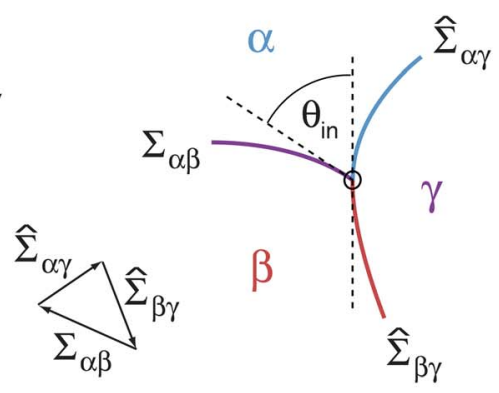

(c)

(d)

Fig. 7 (a) Schematic shape of a vesicle membrane (blue/red) enclosing two liquid droplets of $\alpha$ and $\beta$ phase immersed in the exterior liquid $\gamma$ as observed by conventional optical microscopy. The system contains three surfaces: the $\alpha \beta$-interface (purple) between the two droplets, as well as two different membrane segments that separate (i) the $\alpha$ droplet and (ii) the $\beta$ droplet from the exterior $\gamma$ phase. The three arrows are tangential to these three surfaces along the contact line $(\odot)$, where they define the three effective contact angles $\theta_{\alpha}, \theta_{\beta}$, and $\theta_{\gamma}$ with $\theta_{\alpha}+\theta_{\beta}+\theta_{\gamma}=$ $2 \pi$; (b) the three arrows represent three tensions: the interfacial tension $\Sigma_{\alpha \beta}$ as well as the two membrane tensions $\hat{\Sigma}_{\alpha \gamma}$ and $\hat{\Sigma}_{\beta \gamma}$; (c) because the three tensions balance each other in mechanical equilibrium, they form a triangle, which implies that any chosen tension cannot exceed the sum of the two other tensions; and (d) enlarged view of the vicinity of the contact line: the membrane bends smoothly and the two membrane segments have a common tangent plane (vertical broken line). The angle between this common tangent plane and the plane tangential to the $\alpha \beta$-interface represents the intrinsic contact angle $\theta_{\text {in }} \cdot{ }^{47}$

Both total tensions involve the same auxiliary tension $\Sigma$ since the areas $A_{\alpha \gamma}$ and $A_{\beta \gamma}$ of the two membrane segments can vary and adapt to the volumes of the two liquid droplets as long as the total membrane area is conserved.** In ref. 11 and 47 , two auxiliary tensions were used which is misleading from a conceptual point of view even though it does not affect the final results.

Second, the two membrane segments and the $\alpha \beta$ interface meet along the threephase contact line with effective contact angles $\theta_{\alpha}, \theta_{\beta}$, and $\theta_{\gamma}$ as defined in Fig. 7(a,b). This contact angles are related to the different tensions via the triangle shown in Fig. 7(c). For three interfacial tensions, this triangle is known as the Neumann triangle. ${ }^{56}$ As a consequence, the tensions and the effective contact angles satisfy the relations:

$$
\begin{aligned}
& \Sigma_{\alpha \beta}+\hat{\Sigma}_{\alpha \gamma} \cos (\theta \alpha)+\hat{\Sigma}_{\beta \gamma} \cos (\theta \beta)=0, \\
& \hat{\Sigma}_{\alpha \gamma}+\Sigma_{\alpha \beta} \cos (\theta \alpha)+\hat{\Sigma}_{\beta \gamma} \cos (\theta \gamma)=0,
\end{aligned}
$$

and

$$
\hat{\Sigma}_{\beta \gamma}+\Sigma_{\alpha \beta} \cos \left(\theta_{\beta}\right)+\hat{\Sigma}_{\alpha \gamma} \cos \left(\theta_{\gamma}\right)=0,
$$

two of which are linearly independent and determine the two tension ratios $\hat{\Sigma}_{\alpha \gamma} / \Sigma_{\alpha \beta}$ and $\hat{\Sigma}_{\beta \gamma} / \Sigma_{\alpha \beta}$ by the simple relations:

** In contrast, a vesicle membrane that undergoes phase separation into two intramembrane domains for fixed membrane composition involves two auxiliary tensions, which are conjugate to the fixed areas of the two intramembrane domains. 


$$
\frac{\hat{\Sigma}_{\alpha \gamma}}{\Sigma_{\alpha \beta}}=\frac{\sin \left(\theta_{\beta}\right)}{\sin \left(\theta_{\gamma}\right)}
$$

and

$$
\frac{\hat{\Sigma}_{\beta \gamma}}{\Sigma_{\alpha \beta}}=\frac{\sin \left(\theta_{\alpha}\right)}{\sin \left(\theta_{\gamma}\right)} .
$$

Thus, by measuring the interfacial tension $\Sigma_{\alpha \beta}$ and the effective contact angles, one can obtain the total membrane tensions $\hat{\Sigma}_{\alpha \gamma}$ and $\hat{\Sigma}_{\beta \gamma}$, a procedure that was successfully used in ref. 11 and 50. Furthermore, combining the two relations (71) and (72) with the decompositions $\hat{\Sigma}_{\alpha \gamma}=\Sigma+\sigma_{\alpha \gamma}$ and $\hat{\Sigma}_{\beta \gamma}=\Sigma+\sigma_{\beta \gamma}$ of the two membrane tensions, it also follows that:

$$
\frac{\sigma_{\alpha \gamma}}{\Sigma_{\alpha \beta}}-\frac{\sigma_{\beta \gamma}}{\Sigma_{\alpha \beta}}=\frac{\sin \left(\theta_{\beta}\right)}{\sin \left(\theta_{\gamma}\right)}-\frac{\sin \left(\theta_{\alpha}\right)}{\sin \left(\theta_{\gamma}\right)} .
$$

\subsection{Once again: tensions dominated by spontaneous tensions}

As mentioned, the experiments in ref. 11 also showed that the $\alpha \gamma$-membrane segment in contact with the PEG-rich $\alpha$ phase was connected to many nanotubes protruding into the PEG-rich phase. Since the $\alpha \gamma$-membrane formed a spherical cap, we can directly apply the analysis of the previous section and conclude that the auxiliary tension $\Sigma$ and the spontaneous tension $\sigma_{\alpha \gamma}$ are related via

$$
\Sigma \approx-\frac{R_{\mathrm{cy}}}{R_{\mathrm{sp}}} \sigma_{\alpha \gamma}=-\frac{R_{\mathrm{cy}}}{R_{\mathrm{sp}}} 2 \kappa_{\alpha \gamma} m_{\alpha \gamma}^{2}
$$

as follows from (63). For the PEG-dextran system studied in ref. 11, the spherical curvature radius $R_{\mathrm{sp}}$ was measured to be about $40 \mu \mathrm{m}$, whereas the tube radius was estimated to be about $200 \mathrm{~nm}$ from the theoretical analysis. Thus, for this system, one has $\Sigma \simeq-\sigma_{\alpha \gamma} / 400$ and the total tension

$$
\hat{\Sigma}_{\alpha \gamma}=\Sigma+\sigma_{\alpha \gamma} \approx \sigma_{\alpha \gamma}=2 \kappa_{\alpha \gamma} m_{\alpha \gamma}^{2}
$$

to an excellent approximation. It now follows from (71) that:

$$
\frac{\sigma_{\alpha \gamma}}{\Sigma_{\alpha \beta}} \approx \frac{\sin \left(\theta_{\beta}\right)}{\sin \left(\theta_{\gamma}\right)}
$$

and from (73) that

$$
\frac{\sigma_{\beta \gamma}}{\Sigma_{\alpha, \beta}} \approx \frac{\sin \left(\theta_{\alpha}\right)}{\sin \left(\theta_{\gamma}\right)}
$$

which implies that

$$
\hat{\Sigma}_{\beta \gamma}=\Sigma+\sigma_{\beta \gamma} \approx \sigma_{\beta \gamma}=2 \kappa_{\beta \gamma} m_{\beta \gamma}^{2} .
$$

A combination of the two relations (76) and (77) leads to:

$$
\frac{\sigma_{\alpha \gamma}}{\sigma_{\beta \gamma}}=\frac{\kappa_{\alpha \gamma} m_{\alpha \gamma}^{2}}{\kappa_{\beta \gamma} m_{\beta \gamma}^{2}} \approx \frac{\sin \left(\theta_{\beta}\right)}{\sin \left(\theta_{\alpha}\right)},
$$

which provides a simple and intriguing relation between the spontaneous tensions of the two membrane segments and the effective contact angles as visible in the optical microscope. 


\subsection{Intrinsic contact angle in the presence of spontaneous curvature}

When viewed with optical resolution, the membrane shape exhibits a kink along the contact line as in Fig. 7(a). Because of this kink, one can measure the effective contact angles $\theta_{\alpha}, \theta_{\beta}$, and $\theta_{\gamma}$ displayed in this figure. However, if the membrane shape had a kink that persisted to smaller length scales, its bending energy would become infinite. Therefore, when viewed with higher resolution, the membrane should be smoothly curved as in Fig. 7(d). ${ }^{47}$ The $\alpha \gamma$ - and the $\beta \gamma$-segment then have a comment tangent plane along the contact line. The angle between this common tangent plane and the plane tangential to the $\alpha \beta$-interface represents the intrinsic contact angle $\theta_{\text {in }}$.

Since both membrane segments are assumed to have uniform composition and, thus, uniform elastic parameters, the membrane tensions $\hat{\Sigma}_{\alpha \gamma}$ and $\hat{\Sigma}_{\beta \gamma}$ as defined by (66) and (67) are constant within the corresponding membrane segments up to the contact line. Inspection of Fig. 7(d) then shows that the force balance at the contact line has the simple form:

$$
\Sigma_{\alpha \beta} \cos \left(\theta_{\text {in }}\right)=\hat{\Sigma}_{\beta \gamma}-\hat{\Sigma}_{\alpha \gamma}=\sigma_{\beta \gamma}-\sigma_{\alpha \gamma},
$$

where corrections arising from the contact line tension have been ignored. The contact angle relation (80) looks like the well-known Young equation for liquid droplets at planar surfaces but it describes a completely different situation, since the intrinsic tensions $\sigma_{\alpha \gamma}$ and $\sigma_{\beta \gamma}$ depend on the elastic properties of the two membrane segments. $\dagger \dagger$

Furthermore, inserting the tension ratios (71) and (72) into the contact angle relation (80), all three tensions can be eliminated, and one obtains the purely geometric relation:

$$
\cos \theta_{\text {in }}=\frac{\sin \theta_{\alpha}-\sin \theta_{\beta}}{\sin \theta_{\gamma}}=\frac{\sin \theta_{\alpha}-\sin \theta_{\beta}}{\sin \left(2 \pi-\theta_{\alpha}-\theta_{\beta}\right)} .
$$

Using this latter relation, the intrinsic contact angle can be easily calculated from the measured values of the effective contact angles $\theta_{\alpha}, \theta_{\beta}$, and $\theta_{\gamma}$ without recourse to the tensions.

The relation (81) was first derived in ref. 47 under the simplifying assumption that the membrane segments have no spontaneous curvature. However, without such a curvature, the $\alpha \gamma$-segment of the vesicle membrane could not form the experimentally observed nanotubes as explained in ref. 11. The derivation of the relation (81) as described here shows that this relation is also valid if the $\alpha \gamma$-and $\beta \gamma$-segments of the vesicle membrane have different spontaneous curvatures and/or different bending rigidities.

\section{Summary and outlook}

In this paper, the spontaneous tubulation of lipid bilayers and other biomembranes was studied theoretically. It was shown that adsorption processes can induce a large spontaneous (or preferred) curvature. In all cases studied here and displayed in Fig. 3, the sign of the adsorption-induced spontaneous curvature implies that the membranes prefer to bend away from the aqueous compartment with the higher concentration of adsorbing particles. In general, the opposite direction of bending seems also possible as theoretically found for flexible polymers that are adsorbed to relatively large membrane anchors. ${ }^{57}$ Another case, in which the membranes

$\uparrow \dagger$ Note that (80) would also involve the difference $\Sigma_{\beta \gamma}-\Sigma_{\alpha \gamma}$ if we chose two different auxiliary tensions $\Sigma_{\beta \gamma} \neq \Sigma_{\alpha \gamma}$. Since such a term would make the intrinsic contact angle dependent on the vesicle geometry, we see once more that $\Sigma_{\beta \gamma}=\Sigma_{\alpha \gamma}=\Sigma$ as in (66) and (67). 
should bend towards the compartment with the higher concentration, is provided by proteins or other nanoparticles that have the shape of a hemisphere and adsorb onto the membranes with their spherical surface, but such a system remains to be explored experimentally.

Another mechanism that induces spontaneous curvature is provided by different depletion layers in front of the two membrane surfaces as theoretically studied in ref. 12. Irrespective of its molecular origin, a spontaneous curvature is predicted to generate a spontaneous tension in weakly curved membranes, a tension that can vary over several orders of magnitudes and can be as high as $1 \mathrm{~mJ} \mathrm{~m}$.

For supported lipid bilayers with adsorption-induced spontaneous curvature, a simple criterion for spontaneous tubulation was derived: the bilayers should unbind via the formation of tubules as in Fig. 4(c) when the spontaneous tension exceeds the adhesive strength of the underlying support, see the inequalities in eqn (20) and (45). As mentioned in the introduction, the spontaneous tubulation of multilamellar liposomes has also been observed after the adsorption of BAR domain proteins and other proteins such as epsin. The structure of these liposomes has not been characterized in detail but the adjacent membranes within the multilayer will experience mutual attractive interactions. Thus, the membrane that forms the exterior surface of the multilayer stack experiences the same kind of competition as the supported bilayers: the membrane acquires a spontaneous curvature and associated tension via protein adsorption, and this spontaneous tension again competes with the adhesion towards the adjacent membrane within the multilayer stack.

For giant unilamellar vesicles, it was shown that spherical membrane segments of the vesicle shape as in Fig. 5 can reveal the presence of a large spontaneous tension that balances the osmotic pressure between the interior and exterior compartment, see (65). Such a situation must be present if the vesicles are connected to nanotubes that protrude into the interior compartment as schematically shown in Fig. 6(b,c) and as experimentally discovered for vesicles in contact with two aqueous polymer phases. ${ }^{11,55}$ In general, the spontaneous tension may prevail whenever the vesicles exhibit spherical membrane segments, a situation that is observed frequently, see the examples in Fig. 5, but it is, in general, difficult to identify the spontaneous curvature as the tension-generating mechanism. One very useful exception is provided by vesicles in contact with two aqueous phases as in Fig.7, for which the spontaneous tension can be easily calculated from the effective contact angles via the relations (76) and (77), and the effective contact angles can be directly measured by optical microscopy. Finally, the relation (80) shows that the intrinsic contact angle is determined by the difference of the spontaneous tensions.

One aspect that has not been addressed here for the aqueous two-phase systems is the adsorption of membrane nanotubes onto the interface between the two liquid droplets as observed in ref. 11. This adsorption process is a direct consequence of the partial wetting of the membrane by the two aqueous phases and acts to reduce the effective interfacial tension between the two liquid droplets. The influence of this wetting process will be discussed elsewhere. ${ }^{58}$ In addition, many other aspects of membranes in contact with aqueous two-phase systems remain to be explored. Experimentally, only one such system containing PEG and dextran molecules has been studied so far. The theory described here shows, however, that spontaneous tubulation of giant vesicles will occur for many other polymer or protein solutions as well. Indeed, for an appropriate choice of the polymer or protein concentrations in the interior and exterior compartment, the membranes will prefer to bend away from the interior compartments as a result of the molecular interactions with the polymers or proteins and will then form tubes in order to store their excess areas. Theoretically, it will be rather useful to study the spontaneous tubulation of membranes by computer simulations with molecular resolution in order to estimate the initial energy barriers for this process. 


\section{Acknowledgements}

The theory described here was originally motivated by experimental observations on aqueous two phase systems. I thank Rumiana Dimova, Halim Kusumaatmaja, Yanhong Li, and Yonggang Liu for enjoyable collaborations on these systems as well as Bartosz Różycki and Jaime Agudo for a critical reading of the manuscript.

\section{References}

1 O. Lehmann, Sitzungsberichte der Heidelberger Akademie der Wissenschaften, 1913, Abteilung A, 13. Abhandlung.

2 K. Takei, V. I. Slepnev, V. Haucke and P. D. Camilli, Nat. Cell Biol., 1999, 1, 33-39.

3 B. J. Peter, H. M. Kent, I. G. Mills, Y. Vallis, P. J. G. Butler, P. R. Evans and H. T. McMahon, Science, 2004, 303, 495-499.

4 K. Farsad, N. Ringstad, K. Takei, S. R. Floyd, K. Rose and P. D. Camilli, J. Cell Biol., 2001, 155, 193-200.

5 Q. Wang, V. A. S. Navarro, G. Peng, E. Molinelli, S. L. Goh, B. L. Judson, K. R. Rajashankar and H. Sondermann, Proc. Natl. Acad. Sci. U. S. A., 2009, 106, $12700-12705$.

6 M. G. J. Ford, I. G. Mills, B. J. Peter, Y. Vallis, G. J. K. Praefcke, P. R. Evans and H. T. McMahon, Nature, 2002, 419, 361-366.

7 Y. A. Domanov and P. K. J. Kinnunen, Biophys. J., 2006, 91, 4427-4439.

8 M. Mally, J. Majhenc, S. Svetina and B. Zeks, Biochim. Biophys. Acta, Biomembr., 2007, 1768, 1179-1189.

9 T. M. Domingues, K. A. Riske and A. Miranda, Langmuir, 2010, 26, 11077-11084.

10 A. Arouni, V. Kiessling, L. Tamm, M. Dathe and A. Blume, J. Phys. Chem. B, 2011, 115, $158-167$.

11 Y. Li, R. Lipowsky and R. Dimova, Proc. Natl. Acad. Sci. U. S. A., 2011, 108, 4731-4736.

12 R. Lipowsky and H. G. Döbereiner, Europhys. Lett., 1998, 43, 219-225.

13 P. Canham, J. Theor. Biol., 1970, 26, 61-81.

14 W. Helfrich, Z. Naturforsch., 1973, 28c, 693-703.

15 E. Evans, Biophys. J., 1974, 14, 923-931.

16 R. Goetz, G. Gompper and R. Lipowsky, Phys. Rev. Lett., 1999, 82, 221-224.

17 W. Bancroft and C. Tucker, J. Phys. Chem., 1927, 31, 1681-1692.

18 L. Miao, U. Seifert, M. Wortis and H.-G. Döbereiner, Phys. Rev. E: Stat. Phys., Plasmas, Fluids, Relat. Interdiscip. Top., 1994, 49, 5389-5407.

19 R. Lipowsky, H. G. Döbereiner, C. Hiergeist and V. Indrani, Phys. A, 1998, 249, 536-543.

20 R. Lipowsky, Europhys. Lett., 1995, 30, 197-202.

21 V. Nikolov, R. Lipowsky and R. Dimova, Biophys. J., 2007, 92, 4356-4368.

22 H. T. McMahon and J. L. Gallop, Nature, 2005, 438, 590-596.

23 M. Breidenich, R. Netz and R. Lipowsky, Europhys. Lett., 2000, 49, 431-437.

24 U. Seifert, K. Berndl and R. Lipowsky, Phys. Rev. A: At., Mol., Opt. Phys., 1991, 44, 1182 1202.

25 L. Miao, B. Fourcade, M. Rao, M. Wortis and R. Zia, Phys. Rev. A: At., Mol., Opt. Phys., 1991, 43, 6843-6856.

26 A. Frost, V. M. Unger and P. D. Camilli, Cell, 2009, 137, 191-196.

27 Y. Rao and V. Haucke, Cell. Mol. Life Sci., 2011, 68, 3983-3993.

28 B. Qualmann, D. Koch and M. M. Kessels, EMBO J., 2011, 30, 3501-3515.

29 M. A. Bates and D. Frenkel, J. Chem. Phys., 2000, 112, 10034-10041.

30 H. G. Döbereiner, O. Selchow and R. Lipowsky, Eur. Biophys. J., 1999, 28, 174-178.

31 R. Machan, A. Miszta, W. Hermens and M. Hof, Chemistry and Physics of Lipids, 2010, 163, 200-206.

32 R. Lipowsky, J. Phys. II, 1992, 2, 1825-1840.

33 F. Jülicher and R. Lipowsky, Phys. Rev. Lett., 1993, 70, 2964-2967.

34 C. Dietrich, L. Bagatolli, Z. Volovyk, N. Thompson, M. Levi, K. Jacobson and E. Gratton, Biophys. J., 2001, 80, 1417-1428.

35 S. Veatch and S. Keller, Biophys. J., 2003, 85, 3074-3083.

36 T. Baumgart, S. Hess and W. Webb, Nature, 2003, 425, 821-824.

37 J. B. de la Serna, J. Perez-Gil, A. Simonsen and L. A. Bagatolli, J. Biol. Chem., 2004, 279, 40715-40722.

38 K. Bacia, P. Schwille and T. Kurzchalia, Proc. Natl. Acad. Sci. U. S. A., 2005, 102, 3272 3277.

39 T. Baumgart, S. Das, W. W. Webb and J. T. Jenkins, Biophys. J., 2005, 89, 1067-1080. 
40 R. Dimova, K. A. Riske, S. Aranda, N. Bezlyepkina, R. L. Knorr and R. Lipowsky, Soft Matter, 2007, 3, 817-827.

41 S. Semrau, T. Idema, L. Holtzer, T. Schmidt and C. Storm, Phys. Rev. Lett., 2008, 100, 088101.

42 Z.-C. Ou-Yang and W. Helfrich, Phys. Rev. A: At., Mol., Opt. Phys., 1989, 39, 5280-5288.

43 S. Komura and R. Lipowsky, J. Phys. II, 1992, 2, 1563-1575.

44 D. Bukman, J. Yao and M. Wortis, Phys. Rev. E: Stat. Phys., Plasmas, Fluids, Relat. Interdiscip. Top., 1996, 54, 5463-5468.

45 M. Staykova, D. P. Holmes, C. Read and H. A. Stone, Proc. Natl. Acad. Sci. U. S. A., 2011, 108, 9084-9088.

46 U. Seifert and R. Lipowsky, Phys. Rev. A: At., Mol., Opt. Phys., 1990, 42, 4768-4771.

47 H. Kusumaatmaja, Y. Li, R. Dimova and R. Lipowsky, Phys. Rev. Lett., 2009, 103, 238103.

48 E. Evans, Biophys. J., 1980, 31, 425-432.

49 R. Lipowsky, M. Brinkmann, R. Dimova, T. Franke, J. Kierfeld and X. Zhang, J. Phys.: Condens. Matter, 2005, 17, S537-S558.

50 Y. Li, H. Kusumaatmaja, R. Lipowsky and R. Dimova, J. Phys. Chem. B, 2012, 116, 1819 1823.

51 Y. Li, R. Lipowsky and R. Dimova, J. Am. Chem. Soc., 2008, 130, 12252-12253.

52 M. S. Long, A. S. Cans and C. D. Keating, J. Am. Chem. Soc., 2008, 130, 756-762.

53 M. S. Long, C. D. Jones, M. R. Helfrich, L. K. Mangeney-Slavin and C. D. Keating, Proc. Natl. Acad. Sci. U. S. A., 2005, 102, 5920-5925.

54 Y. Li, PhD thesis, University of Potsdam, 2008.

55 R. Dimova and R. Lipowsky, Soft Matter, 2012, 8, 6409.

56 J. Rowlinson and B. Widom, Molecular Theory of Capillarity, Clarendon Press, Oxford, 1989.

57 M. Breidenich, R. Netz and R. Lipowsky, Eur. Phys. J. E: Soft Matter Biol. Phys., 2001, 5, $403-414$.

58 H. Kusumaatmaja, Y. Liu, R. Dimova and R. Lipowsky, in preparation, 2012. 